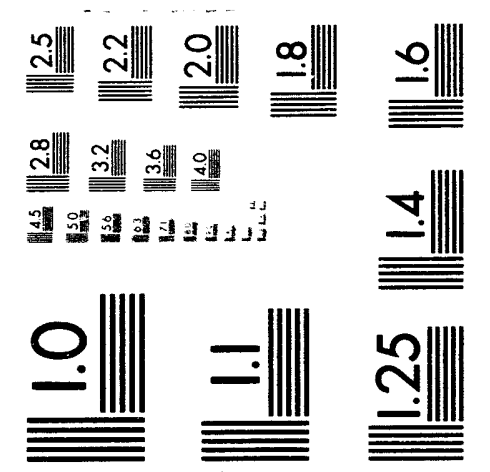




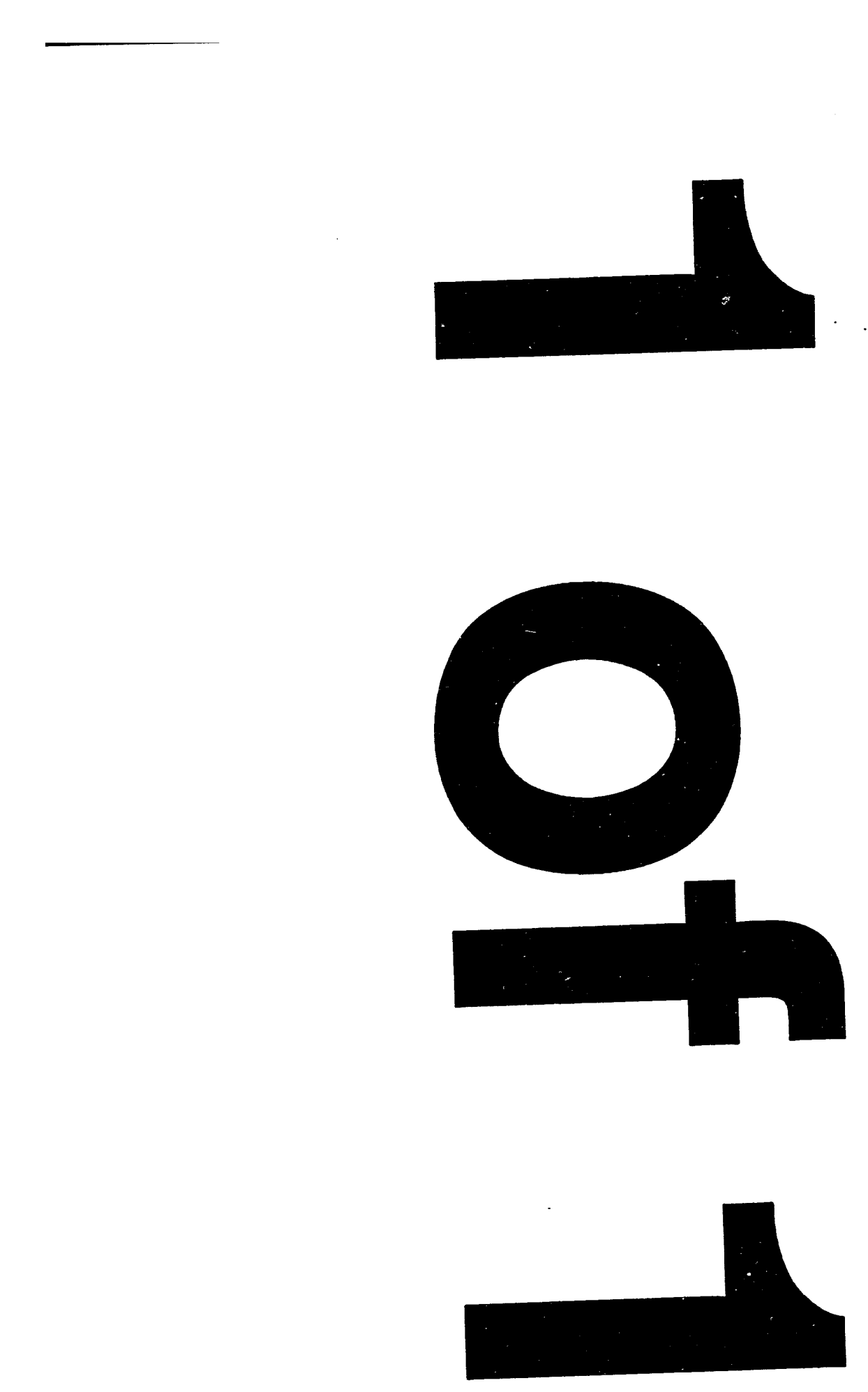


Engineering Physics and Mathematics Division

Mathematical Sciences Section

\title{
NETLIB SERVICES AND RESOURCES
}

\author{
Shirley V. Browne ${ }^{\dagger}$ \\ Jack J. Dongarra $†$ \\ Stan C. Green ${ }^{\dagger}$ \\ Keith Moore ${ }^{\dagger}$ \\ Thomas H. Rowan $\ddagger$ \\ Reed C. Wade $\dagger$ \\ $\dagger$ Department of Computer Science \\ University of Tennessee \\ 107 Ayres Hall \\ Knoxville, TN 37996-1301 \\ $\ddagger$ Mathematical Sciences Section \\ Oak Ridge National Laboratory \\ P.O. Box 2008, Bldg. 6012 \\ Oak Ridge, TN 37831-6367
}

Date Published: April 1994

Research was supported by the National Science Foundation under Grant. No. ASC-9103853, by the Advanced Research Projects Agency under contract DAAL03-92-G-0284 administered by the Army Research Office, and by the Applied Mathematical Sciences Research Program of the Office of Energy Research, U.S. Department of Energy.

Prepared by the

Oak Ridge National Laboratory

Oak Ridge, Tennessee 37831

managed by

Martin Marietta Energy Systems, Inc.

for the

U.S. DEPARTMENT OF ENERGY

under Contract No. DE-AC05-84OR21400 


\section{Contents}

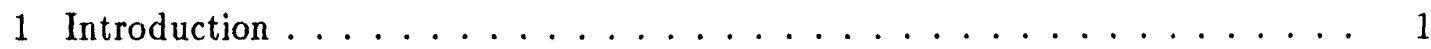

2 Repository Contents .................... 3

2.1 Software and Documents . . . . . . . . . . . . . . 3

2.1.1 Software and Document Submissions . . . . . . . . . . . 3

2.2 Performance Database . . . . . . . . . . . . . . . . . 3

2.3 Conferences Database ... . . . . . . . . . . . . . . 4

2.4 Numerical Analysis Net (NA-NET) . . . . . . . . . . . . . . 4

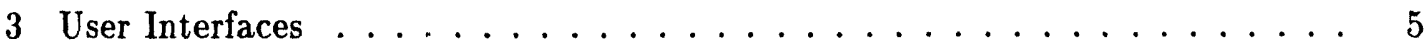

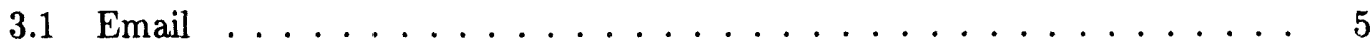

3.1 .1 Support Addresses . . . . . . . . . . . . . 5

3.1.2 Email Interface to the Netlib Repository . . . . . . . . . . . . 5

3.1.3 Email Interface to NA-NET . . . . . . . . . . . . 6

3.1.4 Email Interface to the NA-NET Whitepages . . . . . . . . . 8

$3.2 \mathrm{X}$ Windows Interface - the Xnetlib Client . . . . . . . . . . . . 10

3.2.1 Acquiring the Xnetlib Software . . . . . . . . . . . . 11

3.2 .2 System Rsquirements . . . . . . . . . . . . . 11

3.2 .3 Building Xnetlib . . . . . . . . . . . . . . 12

3.2.4 Xnetlib Man Page and Quick Reference Card . . . . . . . . . . 12

3.2.5 Xnetlib Client Program Operation ... . . . . . . . . . 13

3.2 .6 Command Line Options . . . . . . . . . . . . . . . 16

3.2 .7 Application Defaults File . . . . . . . . . . . . 16

3.2 .8 Support Address . . . . . . . . . . . . . . . . 16

3.3 Netlibget, a Command-line TCP/IP Client . . . . . . . . . . . 17

3.4 Anonymous Access . . . . . . . . . . . . . . . . . 18

3.4.1 Instructions for Anonymous FTP . . . . . . . . . . . 18

3.4.2 Instructions for Anonymous RCP and RSH . . . . . . . 18

3.5 Access via Gopher . . . . . . . . . . . . . . . . . 18

4 Future Plans . . . . . . . . . . . . . . . . . . . . . 19

5 Netlib Repository Setup and Maintenance . . . . . . . . . . . . . . 20

5.1 Netlib Index File Format. . . . . . . . . . . . . . . . . . . . 20

5.2 Repository Replication in Netlib . . . . . . . . . . . . . 22

6 Email Netlib Server Installation . . . . . . . . . . . . . . . . . . 23

6.1 Acquiring and Installing the Netlib Software . . . . . . . . . . . . 23

7 NA-NET Database Setup and Maintenance . . . . . . . . . . . 25

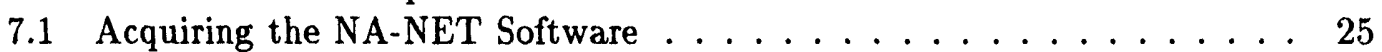

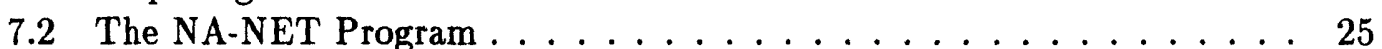

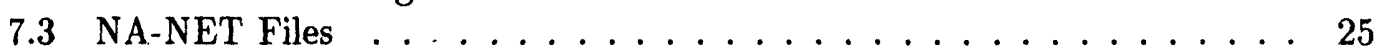

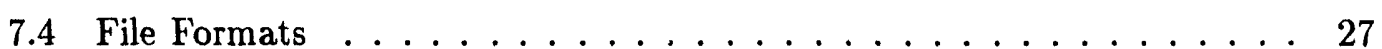

7.5 NA-NET Source Files . . . . . . . . . . . . . 28

7.6 Database Changes, Backups, and Cron Jobs . . . . . . . . . . 28 
7.7 Sending to Digest Subscribers . . . . . . . . . . . . . . . . . . . . 29

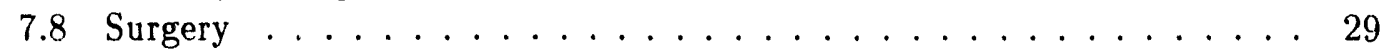

8 Installation and Customization of the Xnetlib Client . . . . . . . . . . . 29

8.1 Acquiring the Xnetlib Software . . . . . . . . . . . . . . . . . . . 29

8.2 System Requirements . . . . . . . . . . . . . . . . . 30

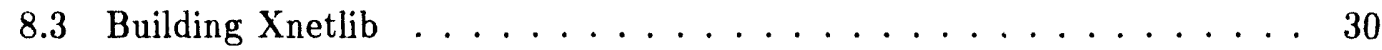

8.4 Customization of Xnetlib $\ldots \ldots \ldots \ldots \ldots$

8.4 .1 X Resources . . . . . . . . . . . . . . . . 31

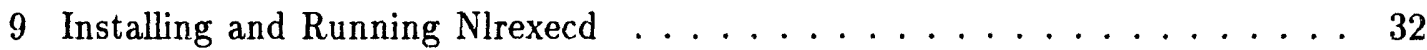

9.1 Acquiring the Nlrexecd Software . . . . . . . . . . . . 32

9.2 System Requirements . . . . . . . . . . . . . . . 32

9.3 Building Nlrexecd . . . . . . . . . . . . . . . . 32

9.4 Services and Protocol . . . . . . . . . . . . . . . 32

9.4.1 Adding a Service to Nlrexecd . . . . . . . . . . . . . 33

9.4 .2 Reserved Service Names . . . . . . . . . . . . . . . 34

9.5 Command Line Options . . . . . . . . . . . . . . . . 35

9.6 Keyword and Database Lookup Services . . . . . . . . . . . . 35

9.6 .1 Keyword Lookup . . . . . . . . . . . . . . . . . 35

9.6.2 Latent Semantic Indexing . . . . . . . . . . . . 35

9.6.3 Whois Service..................... . . 35

9.6.4 Performance Database Service . . . . . . . . . . . . . 36

9.6.5 Conference Database Service . . . . . . . . . . . . 36

10 Anonymous FTP Server for Netlib . . . . . . . . . . . . . . 36

11 Netlib Anonymous RCP Implementation . . . . . . . . . . . . . 38

11.1 "anon" Account . . . . . . . . . . . . . . . . 38

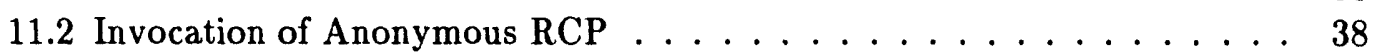

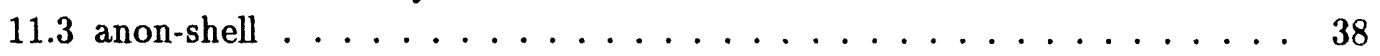

11.4 Modified $r c p$ and 1 s commands . . . . . . . . . . . . 39

11.5 Locations of files . . . . . . . . . . . . . . . . 39

A Netlib Sites ... . . . . . . . . . . . . . . . . 40

A.1 Sites Mirroring the Netlib Repository . . . . . . . . . . . . . . 40

A.2 Some Sites Using the Netlib Email Server to Distribute Other Types of

Software . . . . . . . . . . . . . . . . . . 40

2 References ........................... . . 40 


\title{
NETLIB SERVICES AND RESOURCES
}

\author{
Shirley V. Browne \\ Jack J. Dongarra \\ Stan C. Green \\ Keith Moore \\ Thomas H. Rowan \\ Reed C. Wade
}

\begin{abstract}
The Netlib repository, maintained by the University of Tennessee and Oak Ridge National Laboratory, contains freely available software, documents, and databases of interest to the numerical, scientific computing, and other communities. This report includes both the Netlib iser's Guide and the Netlib System Manager's Guide, and contains information about Netlib's databases, interfaces, and system implementation. The Netlib repository's databases include the Performance Database, the Conferences Database, and the NA-NET mail forwarding and Whitepages Databases. A variety of user interfaces enable users to access the Netlib repository in the manner most convenient and compatible with their networking capabilities. These interfaces include the Netlib email interface, the Xnetlib X Windows client, the netlibget command-line TCP/IP client, anonymous FTP, anonymous RCP, and gopher.
\end{abstract}




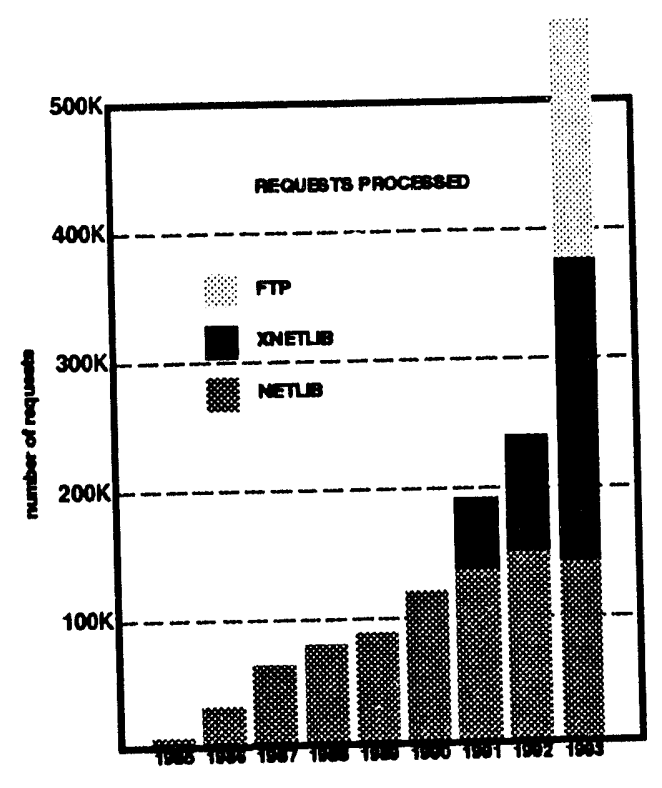

Figure 1: Netlib Requests

\section{Introduction}

Netlib began services in 1985 to fill a need for cost-effective, timely distribution of high-quality mathematical software to the research community. Netlib sends, by return electronic mail, requested routines together with subsidiary routines and any requested documents or test programs supplied by the software authors [5]. Xnetlib, a recently developed interactive tool for software and document distribution [4], uses an X Window interface and TCP/IP connections to allow users to receive replies to their requests within a matter of seconds. The interface provides a number of modes and searching mechanisms to facilitate searching through a large distributed collection of software and documents. The netlibget command-line interface and anonymous FTP and RCP provide services to users who do not need a sophisticated interface. Figure 1 shows the growing number of requests for Netlib services.

A new hypertext/hypermedia version of Xnetlib, currently under development, will interoperate with other information services such as gopher, WAIS, and World Wide Web. It will incorporate a new type of executable document, called an active object, that will greatly enhance the flexibility and adaptability of Xnetlib by allowing runtime binding of functionality.

Although the original focus of the Netlib repository was on mathematical software, the collection has grown to include other software (such as networking tools and tools for visualization of multiprocessor performance data), technical reports and papers, a Whitepages Database, benchmark performance data, and information about conferences and meetings. The number of Netlib servers has grown from the original two, at Oak Ridge National Laboratory (initially at Argonne National Laboratory) and AT\&T Bell Laboratories, to several sites worldwide, including servers in Norway, the United Kingdom, Australia, Japan, and Taiwan. A mirroring mechanism keeps the repository 
contents at the different sites consistent on a daily basis. This mechanism provides redundancy in case of computer or network failures, shares the workload, and broadens human contacts for identifying software to add to the collection.

Netlib differs from other publicly available software distribution systems, such as Archie, in that the collection is moderated by an editorial board and the software contained in it is widely recognized to be of high quality. The user is assured of getting an up-to-date copy of the master version of the requested software. We log requests so we can send bug reports and updates to users of our software. However, the Netlib repository is not intended to replace commercial software. Commercial software companies provide value-added services in the form of support. Although the Netlib collection is moderated, its software comes with no guarantee of reliability or support. Rather, the lack of bureaucratic, legal, and financial impediments encourages researchers to submit their codes by ensuring that their work will be made available quickly to a wide audience.

Requests for consideration of software and document submissions to Netlib, as well as questions, comments, and problems concerning Netlib, should be sent to the following address:

netlib.maintainersonetlib.ornl.gov 


\section{Netlib User's Guide}

\section{Repository Contents}

\subsection{Software and Documents}

The Netlib repository contains a large collection of high-quality public-domain mathematical software. In addition, the repository contains other material of interest to the scientific computing community, including software documentation, test data, technical papers, and reports. Most of the software is written in Fortran, but programs in other languages, such as $\mathrm{C}$, are also available. Netlib is intended primarily for users who wish to obtain individual routines or pieces of a package. Netlib supports dependency checking, so that all of the routines a particular routine depends on (i.e., calls) can be retrieved with it.

The software is organized as a UNIX directory tree. The subdirectories under the Netlib root are called libraries. Each library contains an index file describing the library contents: the files for the individual routines and any subdirectories the library may have. Software routines from a variety of sources are currently available from Netlib. Some of the libraries Netlib distributes - such as EISPACK, LINPACK, FFTPACK, LAPACK, algorithms from the ACM Transactions on Mathematical Software, and algorithms from the book by Forsythe, Malcolm, and Moler [6] - have long been used as important tools in scientific computation and are widely recognized to be of high quality. The Netlib collection also includes a large number of newer, less well-established codes.

\subsubsection{Software and Document Submissions}

To submit software or documents for inclusion in the Netlib repository, follow the guidelines in /netlib/misc/contrib, which you can obtain either by email as follows:

mail netliboornl.gov

send contrib from misc

or by downloading the contrib file from the misc library using the Xnetlib client. Send your submission to the following address:

netlib.maintainersonetlib.ornl.gov

\subsection{Performance Database}

The Performance Database is a publicly-accessible central repository of performance data for all ranges of machines, from personal computers to supercomputers. It provides an on-line catalog of public-domain computer benchmarks such as the LINPACK Benchmark, Perfect Benchmarks, and the NAS Parallel Benchmarks. The benchmark data are presented in a way that facilitates objective interpretations of machine performance. The Performance Database allows all branches of the computing community to archive performance metrics and makes them readily available to the public. For further details, see [1]. 
The performance data are stored in the performance directory in the Netlib repository. Although it is possible to download performance data using anonymous FTP or RCP, the Performance button on the Xnetlib X Windows client provides browsing and keyword searching mechanisms, as well as formatted display of the data. See Section 3.2 for further information.

\subsection{Conferences Database}

The Conferences Database contains conference and meeting announcements. Although it is possible to download conference descriptions using anonymous FTP or anonymous RCP, use of the Conferences button on the Xnetlib client allows searching separately by title, dates, location, or description keywords, as well as viewing of the results in a window. Furthermore, conferences can be submitted only through the Xnetlib client.

The conference description files are stored in the Netlib repository in the confdb directory. The title, start_date, end_date, location, submitter, and submit_date fields are stored in a relational database, currently Postgres. The contents of the title, location, and description fields are added to a full-text index when a conference is entered into the database. A geographical database handles location name aliases and hierarchical geographical relationships. See Section 3.2 for further information.

\subsection{Numerical Analysis Net (NA-NET)}

NA-NET is a community of numerical analysts and other researchers who communicate through a common email facility. NA-NET is supported by a mail-forwarding database and a Whitepages Database. The mail-forwarding database gives users an easy method of communicating with each other through the use of a uniform email address. This feature avoids the problem of having to remember an individual's specific email address. Mail to an NA-NET member can be addressed to na.<key>@na-net.ornl.gov, where 〈key〉 is the member's NA-NET key. The key is usually the member's last name, possibly prefixed by the first letter of the first name. The mail-forwarding database also serves as the distribution list for the NA-NET News Digest. To join or use NANET, use the email interface to NA-NET. See Section 3.1.3 for further details.

The NA-NET Whitepages is a directory service that allows NA-NET members to find out more information about other members. Anyone can join the Whitepages, but NA-NET members are especially encouraged to join. The Whitepages can be accessed by means of the NA-NET Whitepages email interface, the netlibget command-line TCP/IP client, or the Who button on the Xnetlib client. Currently, the Whitepages can be joined only through the email interface. See Section 3.1.4 for further details.

The SIAM Membership Directory is a separate directory that is copyrighted by the Society for Industrial and Applied Mathematics. Queries to the NA-NET whois service that do not find an entry in the NA-NET Whitepages database are referred to the SIAM list. 


\section{User Interfaces}

\subsection{Email}

Anyone with an email connection to the Internet can access most of the Netlib repository. There are email interfaces to the software and document libraries, and the NANET mail-forwarding and Whitepages Databases. To receive more information about the email interface to Netlib, send a message to netlibøornl.gov with the message body send index. To receive more information about the email interface to NA-NET, send a message to na.helpona-net.ornl.gov.

\subsubsection{Support Addresses}

There are email support addresses for the different Netlib services. Users with comments, questions, or bug reports should send a message to the appropriate support address listed below.

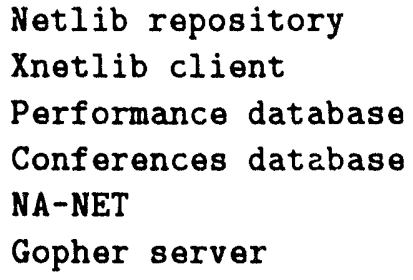

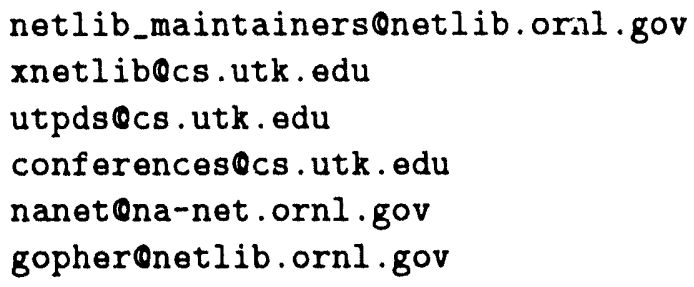

\subsubsection{Email Interface to the Netlib Repository}

\section{Netlib email addresses}

The Internet address netliboornl .gov refers to a gateway machine at Oak Ridge National Laboratory in Oak Ridge, Tennessee. This address should be understood on all the major networks through the normal Domain Name System name resolution.

For access outside the United Slates, you may want to use one of the repositories that mirror the UT/ORNL repository. See Appendix A for a list of these other Netlib sites.

\section{Request syntax}

A valid Netlib email request has a message body that is of one of the following basic forms:

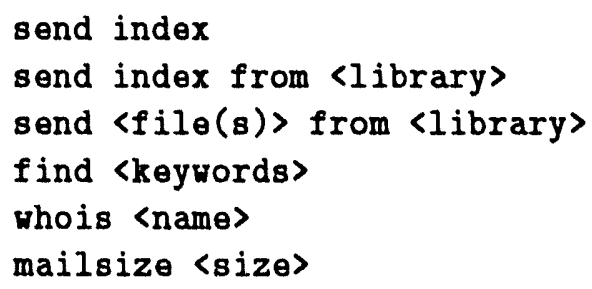

Here are examples of the various kinds of requests. 
* To get the master index for netlib:

send index

* To get the full index for a library:

send index from eispack

* To get a particular routine and all it depends on:

send dgeco from linpack

* To get just the one routine, not subsidiaries:

send only dgeco from linpack

* To get dependency tree, but excluding a subtree:

send dgeco but not dgefa from linpack

* To just tell how large a reply would be, don't actually send the file:

send list of dgeco from linpack

* To get a list of sizes and times of all files in a library:

send directory for benchmark

* To search for somebody in the SIAM membership list:

whois gene golub

* To do a keyword search for Netlib software:

find cubic spline

* To do a bibliographic search:

find schumaker from approximation

find aasen from linalg

* To set the chunk size used for reply:

mailsize $100 \mathrm{k}$

* (optional) End of request:

quit

\subsubsection{Email Interface to NA-NET}

\section{Individual (unicast) messages}

Sending email to an individual NA-NET member is the most frequently used feature of NA-NET. Each NA.NET member has a unique NA-NET name, or key. Mail can be sent to an NA-NET member by addressing it to na.<key>@na-net.ornl.gov, where 〈key> is the member's NA-NET name. The NA-NET name is usually the member's first initial prepended to her last name, the member's last name, or the member's first 
name followed by the first letter of her last name. For example, possible NA-NET names for Joan Smith would be jsmith, smith, and joans.

\section{NA-NET News Digest}

Any mail sent to na.digest@na-net.ornl .gov will be considered for distribution to all members of NA-NET. Once a week, we send out a digest of information contributed by users of NA-NET. The editor of the NA-NET News Digest goes over the messages that have been received, picks out the ones thought to be of general interest to the numerical analysis community, combines them in the News Digest format, and mails the Digest to everyone on the mailing list.

\section{Joining NA-NET}

To join NA-NET, send mail to na.joinena-net.ornl.gov. In the message body, specify the following three fields:

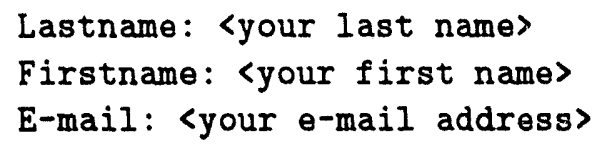

The values can be specified in any order. The subject line of your message will be ignored. An attempt will be made to assign to you a unique NA-NET name consisting of your first initial prepended to your last name, your last name, or your first name followed by the first letter of your last name. If at least one of these keys is not already in use, you will receive a message indicating that your join attempt succeeded and telling you which key has been assigned. If all three of these keys fail to be unique, you will receive an error message indicating that your join attempt failed. In case of failure, send a message to nanetona-net.ornl.gov, and you will be assigned a unique key manually.

\section{Removing membership}

To remove your membership from NA-NET, send mail to na.removenna-net.ornl.gov. In the message body, specify the following two fields:

Lastname: 〈your last name>

Firstname: 〈your first name>

The values can be specified in any order. The subject line of your message will be ignored. NA-NET will send an acknowledgment message to both the deleted address and the address making the request. If more than one entry exists with the same first and last name, you will receive a message indicating that your removal attempt failed. In this case, you can resubmit the removal request with the additional line:

\section{Key: 〈your NA-NET key>}

\section{Changing your email address}


To change your email address in the NA-NET mail-forwarding database, send mail to na.changeona-net.orn!.gov. In the message body, specify the following three fields:

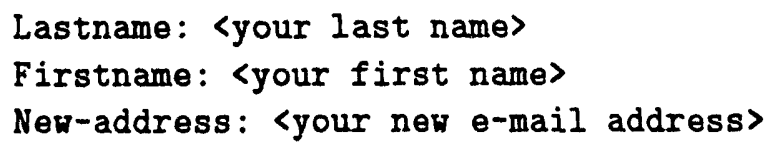

The values can be specified in any order. The subject line of your message will be ignored. An acknowledgment message will be sent to both the old email address as well as the new address informing you that the change has taken place.

If more than one entry e:ists with the same first and last name, you will receive a message indicating that your change attempt failed. In this case, you can resubmit the change request with the additional line:

\section{Key: 〈your NA-NET key>}

\section{Help with NA-NET}

Questions and comments about NA-NET should be addressed to nanetena-net.ornl.gov. Mail sent to na.helpena-net.ornl.gov will receive a reply message describing both NA-NET and the Whitepages.

\section{Current member list}

Mail sent to na.sendlistena-net.ornl.gov will receive a reply message being sent back to you containing the email addresses of all members of NA-NET.

\subsubsection{Email Interface to the NA-NET Whitepages}

\section{Querying the Whitepages Database}

To find out information about a person, send mail to na.whoisena-net.ornl.gov. In the message body or on the subject line specify the person's first name and last name, or just the last name. The order of first name and last name does not matter. For example, to find out more about Jack Dongarra:

mail to: na.whoisena-net.ornl.gov

Subject :

Jack Dongarra

or

mail to: na.whois@na-net.ornl.gov

Subject: jack dongarra

〈null body> 
Keyword searching is also possible. For example, to find out more information about all people who are interested in parallel, send the following message:

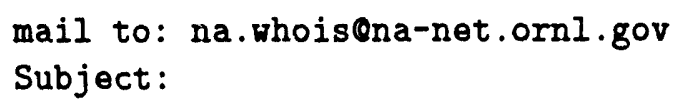

Keyword: parallel

This query does a string search for the pattern parallel on all fields other than the name fields.

As another example, to find out more about all people who live in Knoxville:

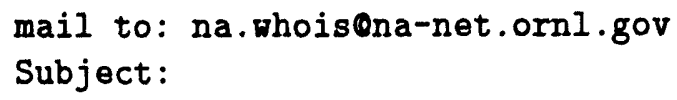

Keyword: Knoxville

This query does a string search on all fields other than the name fields for the pattern Knoxville.

\section{Joining the Whitepages}

To join the NA-NET Whitepages, send mail to na.join-wpena-net.ornl.gov. In the message body, specify the two mandatory fields and as many of the optional fields as you want.

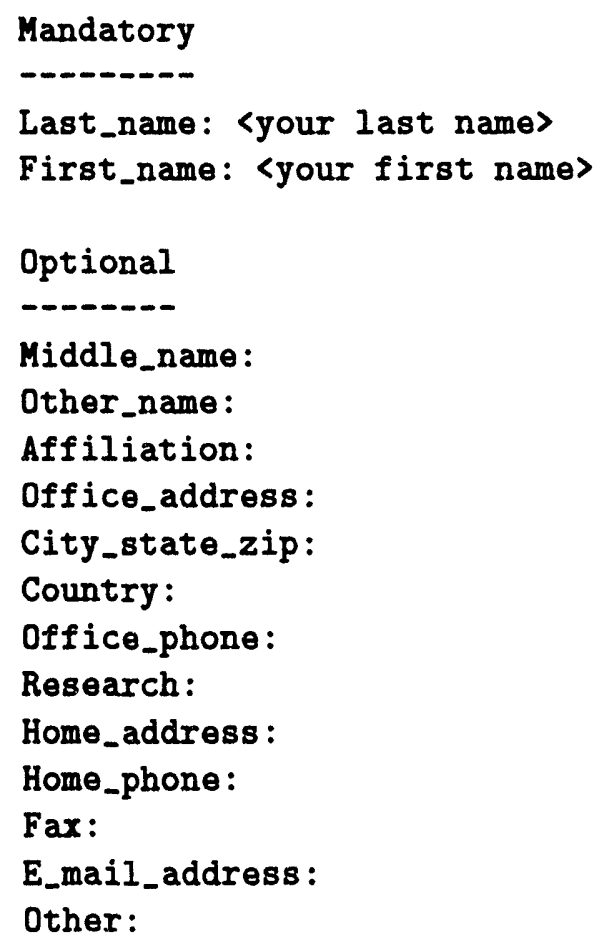


The fields can be specified in any order. The subject line of your message will be ignored. All fields are entered into the database as characters, so spaces can be used for readability. All fields except first_name, last_name, and middle_name can be multiple lines. A multiple-line field ends when the next keyword (e.g., "Country:") is encountered. Each line should end with a carriage return. If your first_name and last_name combination is not unique, send mail to nanetona-net.ornl.gov, and your name will be manually inserted into the Whitepages Database in spite of the duplication. Such duplication will not cause any problems for people querying the Whitepages Database because the database is set up to return information on all people with a given last name, first_name, or combination. An acknowledgment to your join request will be sent back to you confirming that the operation was successful.

\section{Removing your Whitepages entry}

To remove your entry from the NA-NET Whitepages Database, send mail to na.remove-wpena-net.ornl.gov. In the message body, specify the following two fields:

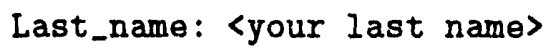

The values can be specified in any order. The subject line of your message will be ignored. An acknowledgment message will be sent to both the address requesting the removal and to the address listed in the Whitepages Database.

\section{Changing fields}

To change the value of a field, to add a field, or to delete a field, send mail to na.change-wpena-net.ornl.gov. In the message body, specify the following two fields:

Last_name: 〈your last name>

First_name: 〈your first name>

plus the fields to be added, changed, or dropped. The fields can be specified in any order. The subject line of your message will be ignored. You can not change your name. If you need to change your name, first remove your entry and then rejoin with the new name. If you wish to clear the value of a field, simply include the field with no value. An acknowledgment message will be sent back to you confirming that the operation was successful.

\section{2. $\mathrm{X}$ Windows Interface - the Xnetlib Client}

$\mathrm{Xnetlib}$ is an X Window System application that provides interactive file access and database query processing from multiple servers through TCP/IP connections. Xnetlib currently provides access to the Netlib software and document repository, the NANET Whitepages Database, the Performance Database, and the Conferences Database. Future releases of Xnetlib will provide additional features, such as access to remote 
execution facilities and interoperability with other information services, such as gopher, WAIS, and World-Wide-Web.

\subsubsection{Acquiring the Xnetlib Software}

To acquire the software for the Xnetlib client send email to netlibcorn?.gov with the line

send xnetlib.shar from netlib

as the body of the message. Netlib will return the file xnetlib.shar by email.

Xnetlib is available by anonymous FTP from netlib2.cs.utk.edu in the xnetlib directory. Both executables and source are available.

To use anonymous FTP to retrieve an executable file, type

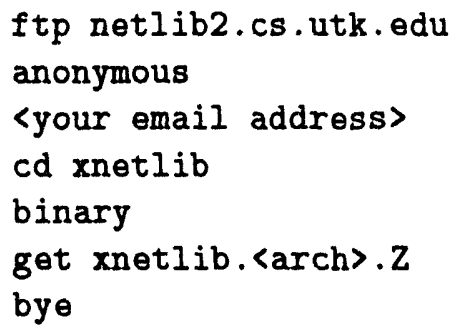

where $<$ arch $>$ is your machine architecture (alpha, hp9000, next, pmax, rs6000, or sun4). To use anonymous FTP to retrieve the Xnetlib source, type

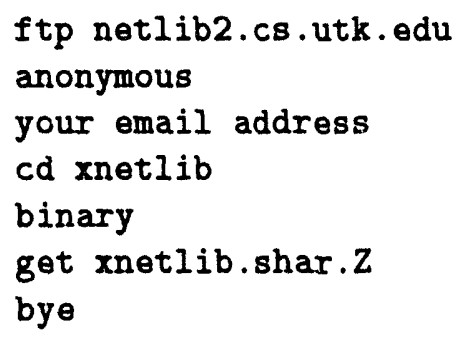

Then move the executable file, named xnetlib to where you want it. See Section 3.2.3 for more information about building Xnetlib.

If you retrieve an executable, you may also wish to retrieve the shar file xnetlib3.4.doc.shar, which contains the Xnetlib man page and quick reference card. These documents are already included in the source code shar file.

\subsubsection{System Requirements}

Xnetlib can be built on nearly any UNIX system. It runs under the X Window System, version 11 , from MIT. It requires release level 4 or greater and the Athena widget libraries as supplied by MIT. 


\subsubsection{Building Xnetlib}

If you retrieved an executable (e.g., xnetlib.sun4), install it by uncompressing it and changing the mode to executable, e.g.,

uncompress xnetlib.sun 4.2

chmod 755 xnetlib.sun4

Xnetlib makes use of the imake facility (via xmkmf) that comes with standard X Windows distributions. A generic Makefile is provided and can be used if imake is not present on your system.

If you retrieved a compressed shar file of the Xnetlib source code, extract the files and build Xnetlib by typing

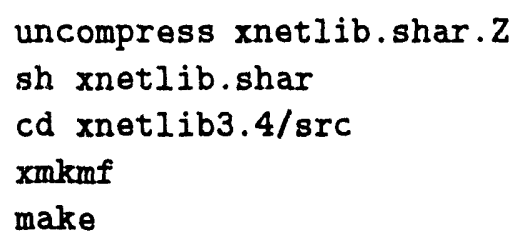

After the executable is built, install it by copying the file xnetlib to an appropriate directory. There is no application defaults file to install.

With imake (xmkmf), type

xmkmf

make

Without imake (xmkmf), first edit Makefile.std to reflect your system characteristics and then type

make -f Makefile.std

xmkmf should be installed on your system as part of the $\mathrm{X}$ distribution. If you get an error on the xmkmf command, check your command search path or talk to your system manager. If you are using an IBM/RS6000, you may need to refer to the information in xnetlib3.4/doc/README. AIXv3.

For further information on system-wide installation of the Xnetlib client and on customization of the Xnetlib client, see the section in the Netlib Manager's Guide on the Xnetlib client (Section 8).

\subsubsection{Xnetlib Man Page and Quick Reference Card}

The source code distribution in the xnetlib3.4/doc directory include the man page for Xnetlib. To view it, cd to this directory and type nroff -man xnetlib.man You may wish to have your system manager install the Xnetlib man page on your system. 


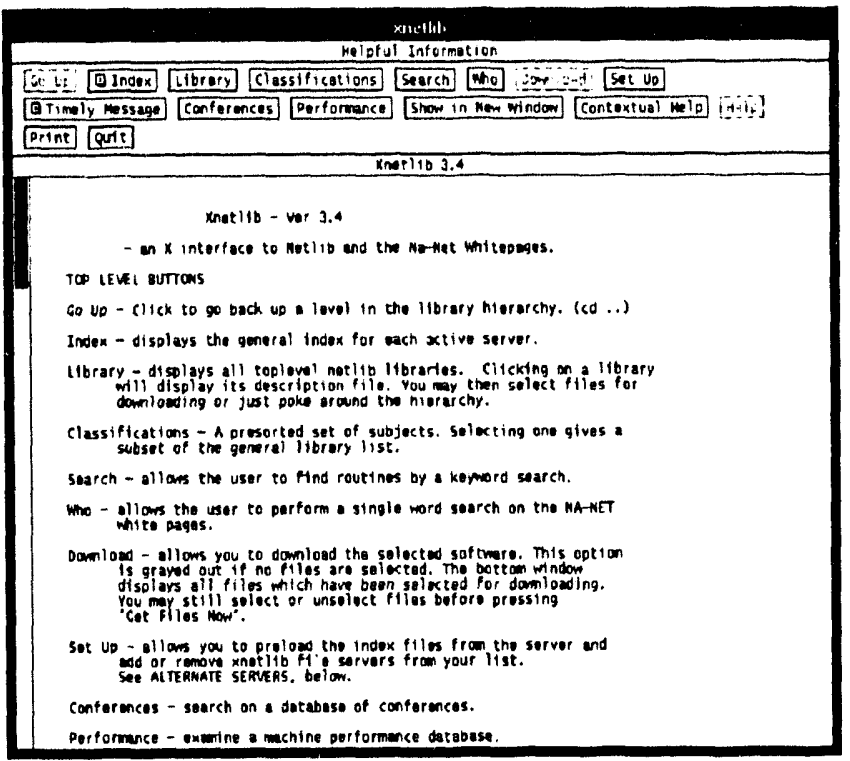

Figure 2: Help Screen

\subsubsection{Xnetlib Client Program Operation}

Start the Xnetlib client program by typing xnetlib. When Xnetlib starts up, it attempts to connect to each server on its list of active hosts. The default active host list is netlib.ornl.gov, netlib.brl.mil and softlib.cs.rice.edu. When these connections have been tested, a window will appear displaying the Xnetlib help screen (Figure 2).

The top row of buttons controls mode changes. In general, Xnetlib reuses the bottom portion of the main window for displays instead of popping up new windows.

Clicking on Library displays the top-level listing of libraries available from the Netlib repository (Figure 3). You can think of this top-level listing as a set of UNIXstyle directories. Clicking on a library name displays a description of the library's contents. For example, clicking on lapack displays the contents of the LAPACK library in library selection mode (Figure 4).

There may be further subdirectories, which are indicated by the folder icon. Files are indicated by the dog-eared page icon. You can select files to be downloaded by clicking on them. Move up the library hierarchy by using the Go Up button and move down by clicking on a library name.

When you have selected one or more files to download from the server, click the Download button to enter download mode (Figure 5). A list of the files you selected will be displayed. You can alter your selections if desired. Click Get Files Now to begin file downloading.

The default directory in which download files are placed is xnlFiles in your home directory. You can change the default directory by clicking on the Download path button. You can choose to have dependency checking either on or off (default is on) by clicking the Dependency checking button. If dependency checking is on, routines 


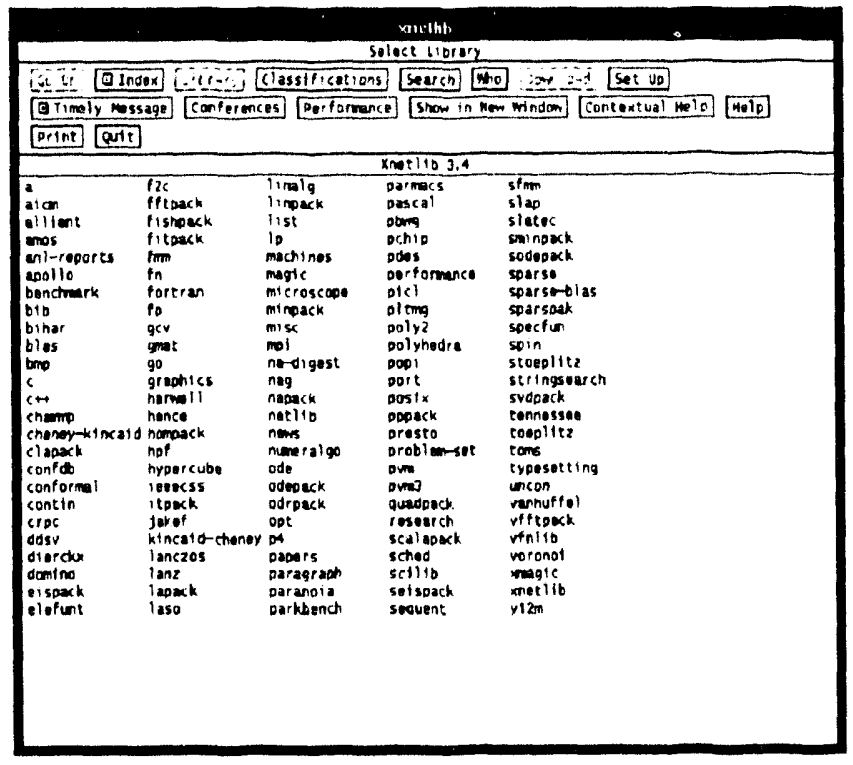

Figure 3: Library List

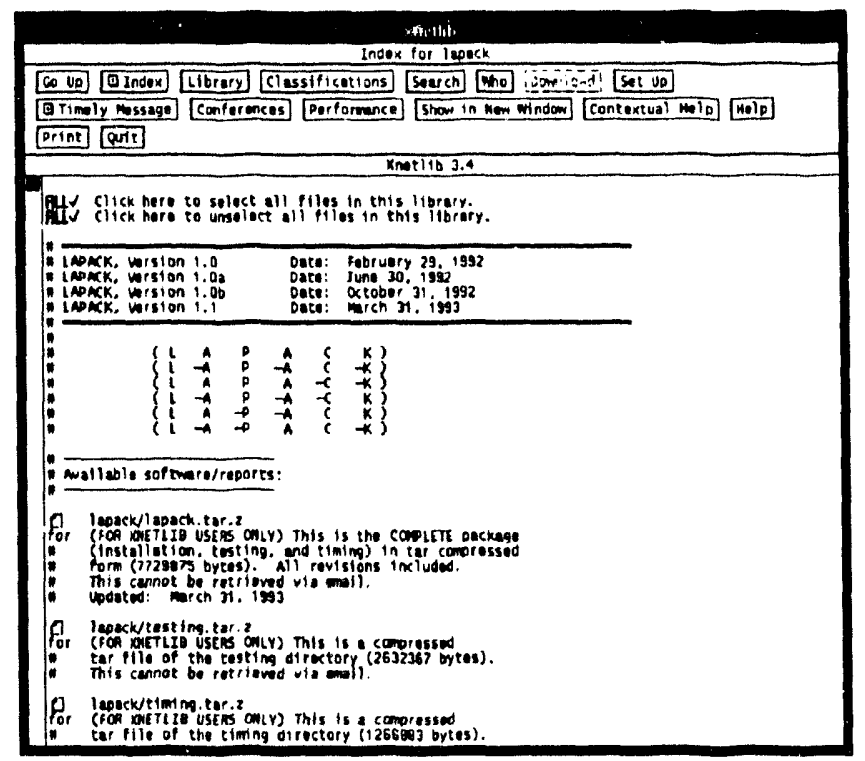

Figure 4: Library Selection Mode 


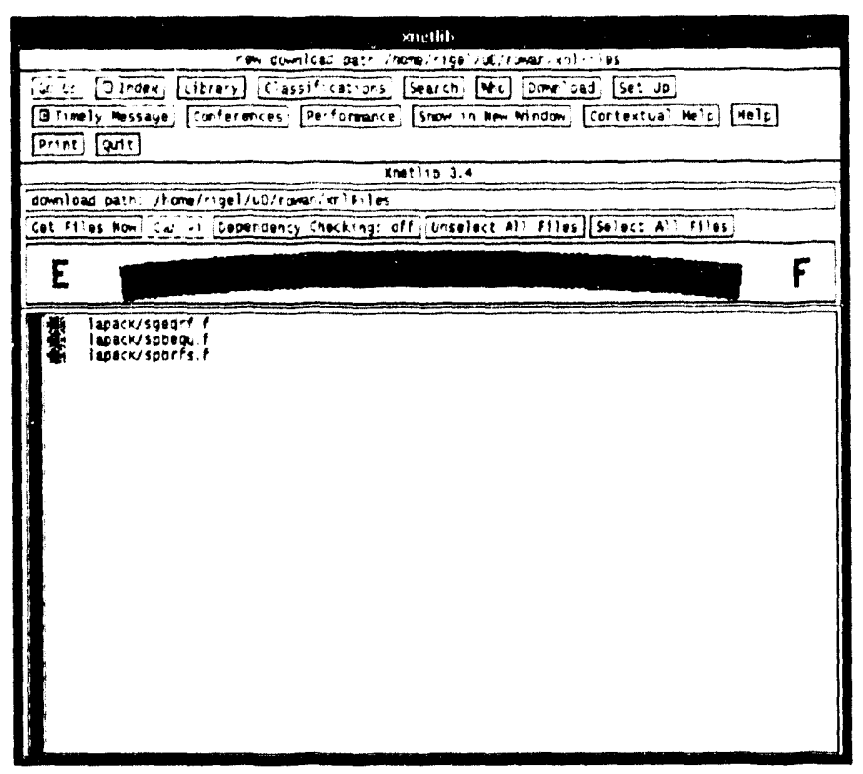

Figure 5: Download Mode

required by the requested file will be appended to the file before it is downloaded.

As you traverse the Netlib tree to examine libraries, the Netlib server at UT/ORNL downloads index files for the libraries to your site. Depending on your network connection, this file transfer may be hardly noticeable or may cause a significant delay. If you prefer to have all the index files loaded at once instead of as you need them, click Set Up, then Press to Check Each Index File. This will check every index file older than indexLifetime (see Section 8.4.1 on Xnetlib X Resources), and will copy from the server any that are missing or changed. To find out how to have several users at your site use one shared collection of index files, instead of several separate collections, see Xnetlib Installation and Customization in the System Manager's Guide (Section $8)$.

Clicking on Classifications lists library topics (Figure 6). The classification is an augmentation of the top level of the GAMS hierarchy. For more information about GAMS, see [2].

Selecting one of these topics displays a subset of the main library list.

Clicking on Search takes you into search mode. Buttons for the various types of searches are displayed, along with an explanation of these search types in the main window. The result of a search is a listing of files, from which you can choose files for downloading.

Clicking on who changes to whois mode and allows you to query the NA-NET Whitepages and SIAM membership list for information about members of these groups.

Clicking on Conferences takes you into the Conferences Database mode. This mode is an experimental interface to a more general database service based on the relational database model. The Conferences Database contains conference and meeting announcements entered by UT staff and by Xnetlib users.

Clicking on Performance takes you into the Performance Database mode. This 


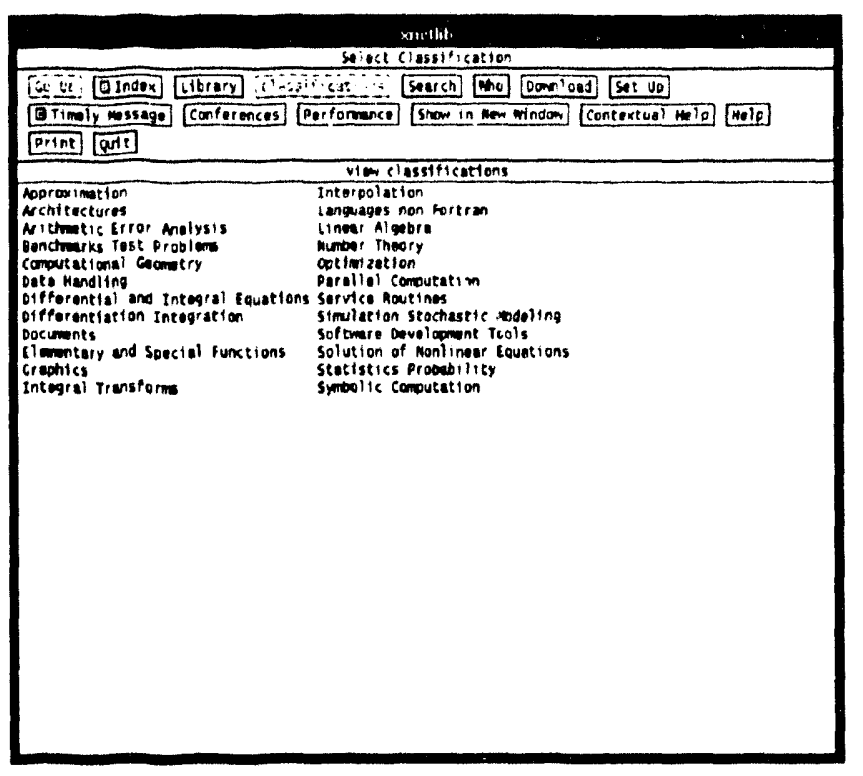

Figure 6: Classifications

mode gives you access to benchmark performance data for a wide range of machines. For more information, see [1].

\subsubsection{Command Line Options}

Xnetlib understands the normal X Toolkit options (-display, -rv, etc.) as well as -help, and those listed in Figure 7 next to the $X$ resources they affect. See Xnetlib Installation and Customization in the System Manager's Guide (Section 8 in this document) for an more complete explanation of these X Resources.

\subsubsection{Application Defaults File}

There is no required application defaults file for Xnetlib. However, you can set application defaults for Xnetlib in your .Xdefaults file in the usual manner. For example, to set the background color for the Xnetlib client to seashell, include the following line in your. Xdefaults file:

xnetlib*background:

seashell

For further information about setting application defaults, see [8].

\subsubsection{Support Address}

Please send questions, comments, suggestions, or bug reports concerning Xnetlib to the following email address:

xnetlibecs.utk.edu 


\begin{tabular}{|l|ll|}
\hline Option & Resource & \\
\hline -dw & dontWarp & no gratuitous cursor motion \\
-nocq & confirmQuit & no confirm on quit \\
-cq & confirmQuit & confirm on quit \\
-showdown & showDownloadAnyway & download button always on \\
-noshowdown & showDownloadAnyway & download button not always on \\
-showwho & showWholnfo & show help in who mode \\
-noshowwho & showWholnfo & don't show help in who mode \\
-pub[lic] & publicData & write index files world writable \\
-nopub[lic] & publicData & don't write index files world writable \\
-dc & depCheck & dependency checking on \\
-nodc & depCheck & dependency checking off \\
-path <path> & xnlDownloadPath & where files are placed \\
-dp <path> & xnlPath & where index files are cached \\
-es <string> & entrySep & entry separator in who mode \\
-pc <string> & printCommand & printing command \\
-life <number of days> & indexLifetime & how long to keep index files \\
-email <email address> & email & your email address for our logs \\
-f[ile] <file name> & hostFile & xnetlib server list \\
\hline
\end{tabular}

Figure 7: Xnetlib options and $\mathrm{X}$ resources

\subsection{Netlibget, a Command-line TCP/IP Client}

Users who do not have $X$ Windows may use netlibget, a TCP/IP command-line interface to the Xnetlib servers. This command-line client is included with the Xnetlib release. To build it from the Xnetlib source, type the following:

make -f Makefile.std netlibget

Running netlibget without any options will display the following usage message:

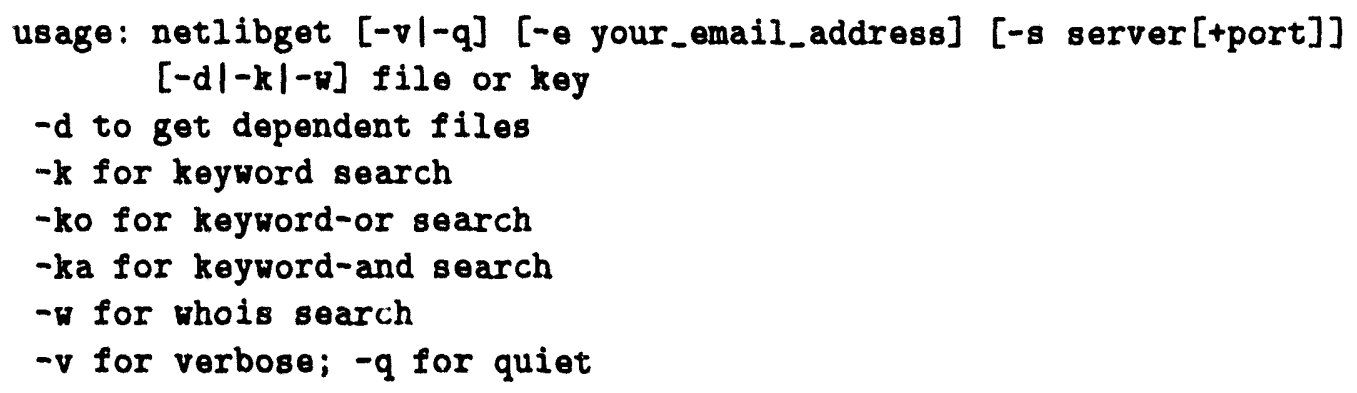

For example, to retrieve dgeco.f from linpack with dependent routines, type the following:

\section{netlibget - d linpack/dgeco.f}

For a keyword-or or keyword-and search, the list of keywords should be enclosed in single quotes. For example, to search for files whose descriptions contain the words condition and number, type the following: 
netlibget -ka 'condition number'

\subsection{Anonymous Access}

For users who do not need the search capabilities provided by Xnetlib, anonymous access in the form of anonymous FTP and anonymous RCP are provided. For more information about anonymous access, try the following:

finger anonenetlib.ornl.gov

\subsubsection{Instructions for Anonymous FTP}

You can use anonymous FTP to retrieve Netlib files. Ftp to netlib2.cs.utk. edu and $\log$ in as anonymous. Use your complete email address as the password.

The Netlib $f t p$ server supports automatic creation of compressed and/or tar'ed

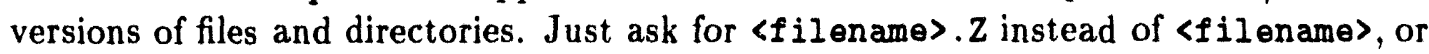
〈directory>.tar. $Z$ instead of each file in the directory, and it gets packaged up on the fly.

\subsubsection{Instructions for Anonymous RCP and RSH}

You can use anonymous RCP to copy files and anonymous RSH to list directories.

1. To copy a particular file to your system, type

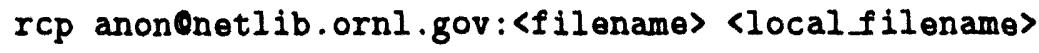

where 〈filename $\rangle$ is the filename in the Netlib repository and 〈local filename〉 is the local file in which you wish to have the data stored on your system.

For example, to retrieve sgetrf. $f$ from lapack, type

rcp anonenetlib.ornl.gov:lapack/sgetrf.f sgetrf.f

2. To list files from a particular directory, type

rsh netlib.ornl.gov -1 anon 1s <directory>

(On some machines, the command is remsh instead of $\mathrm{rsh}$ ).

Normal 18 options work; $18-1$ gets the size, etc. You can use metacharacters by putting the argument to 18 in single quotes.

\subsection{Access via Gopher}

A gopher server is running on a Netlib machine and can be accessed at the address netlib.ornl.gov on port 70. The gopher URL is gopher:

netlib.ornl.gov:70. The Netlib libraries are listed as entries in the top-level menu. 


\section{Future Plans}

We plan to expand the Netlib repository from a handful of mirrored servers to a virtual repository consisting of many servers. All of these servers will be accessible from the Xnetlib client program. The services offered will include access to software, documents, and databases, as well as remote execution facilities. A user will not need to know where a particular service is located but will be able to employ both browsing and searching mechanisms to locate and access the desired service.

Future releases of Xnetlib will provide a means whereby newly inaugurated services using the Xnetlib protocol will not require use of a new version of the Xnetlib application. The special-purpose code describing the window layout and functions will be downloaded at run time from the remote service provider. This run-time binding of functionality will greatly enhance the flexibility and adaptability of Xnetlib.

As the software and document base grows in size and complexity, simple syntactic keyword searching mechanisms, such as those provided by WAIS, are likely to prove inadequate. We plan to add new search mechanisms that incorporate semantics and make use of more structured knowledge representations. 


\section{Netlib System Manager's Guide}

This guide is intended to assist a system administrator in obtaining, installing, and maintaining the files, databases, and client/server software associated with Netlib. It also explains how to set up and maintain the underlying directories and databases.

\section{Netlib Repository Setup and Maintenance}

The Netlib repository consists of a collection of libraries. A library contains a group of routines that can be retrieved individually. The repository is organized on disk as a UNIX directory tree, hereafter called the "Netlib tree". Assume for purposes of this explanation that the root of the Netlib tree is /netlib. Underneath this root are subdirectories for the various libraries. (For example, the EISPACK library is in /netlib/eispack). The library subdirectories contain either further subdirectories or files. Although the Netlib tree can be accessed via anonymous FTP and anonymous RCP and can also be browsed via the Xnetlib client, additional searching capabilities were thought to be desirable. Thus, the Netlib tree has been augmented by the inclusion of index, or description, files. The file /netlib/master/index contains a listing of all the libraries with descriptions. An index file in the subdirectory for each library lists and describes the library contents. The index files are in a format that is intended to be easily parsed by searching tools. Both the Netlib and the Xnetlib servers use the index files as the basis for their searching mechanisms.

A site may wish to replicate its repository contents, in order to achieve greater reliability and take advantage of load balancing. This replication has been carried out at UT/ORNL, where the Netlib tree is duplicated on two machines, one at UT and the other at ORNL. The copy at UT is the master, and the copy at ORNL is the slave. Whenever someone updates the master copy, that person manually forces propagation of the update to the slave copy using a program that runs the rdist command.

Autonomous sites that maintain copies of the same files need a mechanism to keep those copies consistent. Each file has one master copy and some number of slave copies. The site holding the master copy may be different for different files. Netlib has adopted a low overhead repository mirroring scheme, based on checksum files and $\mathrm{ftp}$, that keeps slave copies of files consistent with the master copies. For more information on this repository mirroring mechanism, see [7].

\subsection{Netlib Index File Format}

Netlib is intended to be not just a warehouse but a library, and for that it must have adequate search tools. Each Netlib directory comes with an index file in the format shown below, to promote searchability.

An index file contains one paragraph per file or subdirectory, each separated from the next by an empty line. Each line of the paragraph contains a keyword, then a tab, and finally a corresponding value. If no value is known or applicable, that line is omitted. If all the files in a directory share a value, that line is moved up in the hierarchy to the index file in the parent directory. 
An entry for a regular file starts with the keyword file, followed by the pathname of the file relative to /netlib. An entry for a directory starts with the keyword lib, followed by the pathname of the directory relative to /netlib. In schematic form, the keywords are as follows:

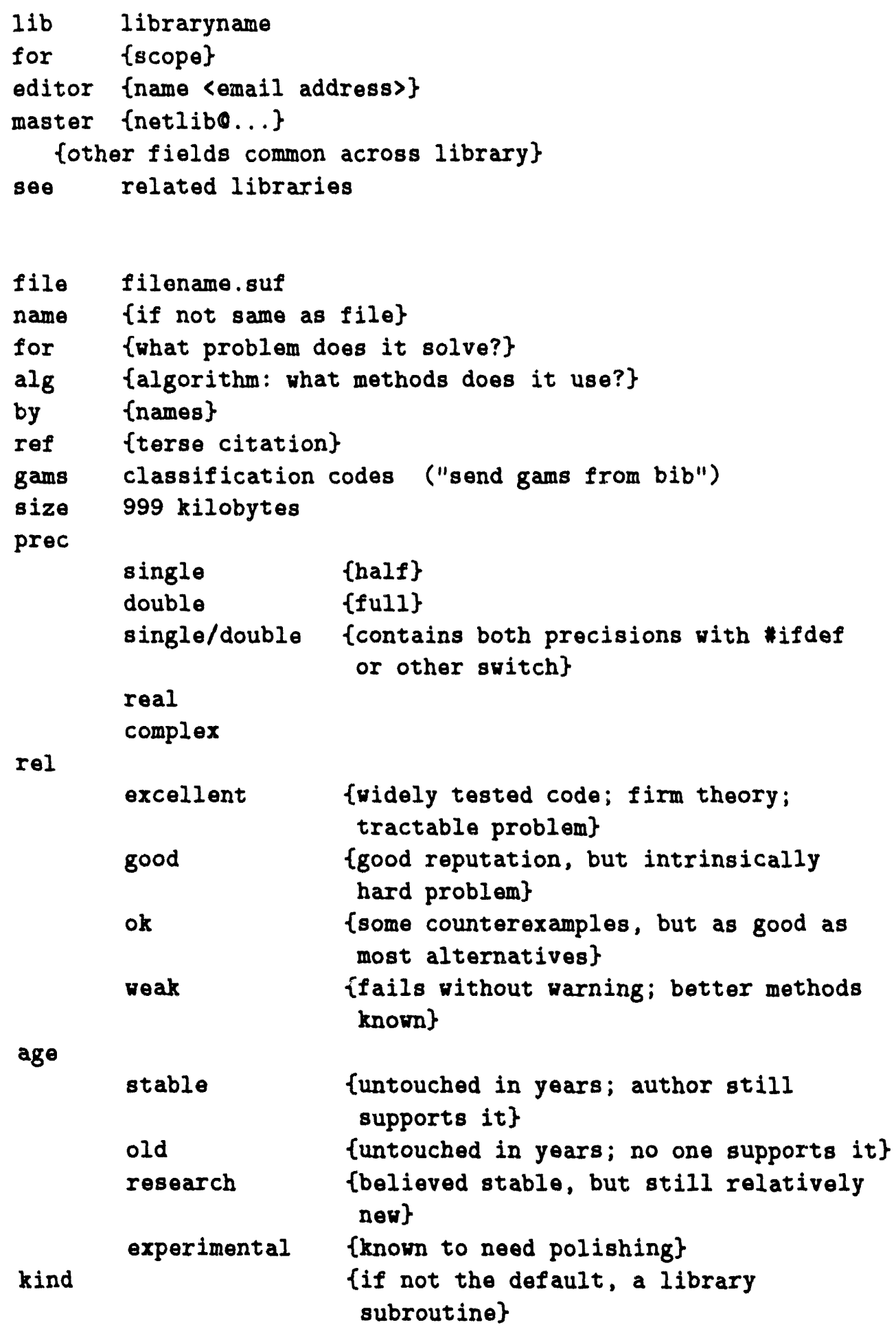




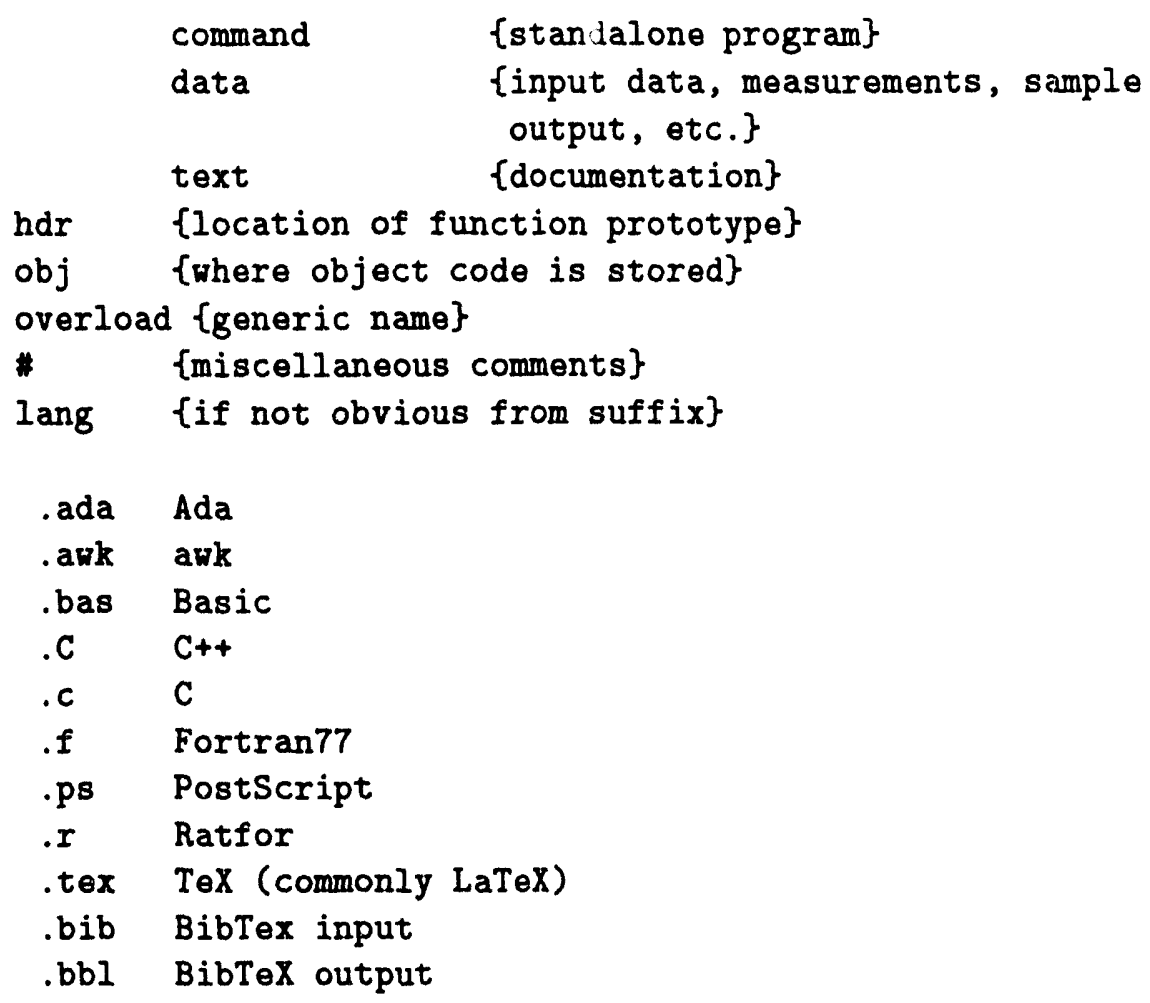

Besides the index file, a directory may also have the files

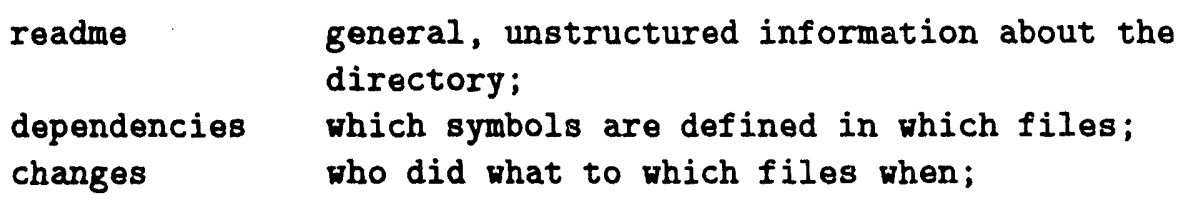

There are two kinds of "test" subdirectories:

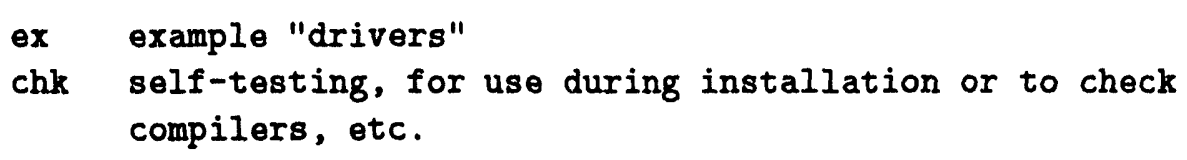

\subsection{Repository Replication in Netlib}

At UT/ORNL, the Netlib repository contents are replicated on two machines. The master copy resides on netlib2.cs.utk.edu, while the slave copy resides on netlib1.epm.ornl.gov. Both machines are registered with the Domain Name System as host addresses for the domain name netlib.ornl.gov. Theoretically, the load balance for Xnetlib requests between the two machines should be about 50/50. The mail preference is currently set up so that netlib1 gets most of the email requests. If netlib1 is down, however, em il requests should get sent to netlib2.

Updates to the repositor; must be made on the master copy on netlib2. When a staff person makes an update, either by installing a new library or by changing or adding files in an existing library, that person is supposed to run a script that logs 
the change and asks him or her whether to propagate the change to netlib1. When the person answers yes, the update is propagated by running the rdist command. A nightly cron job runs an rdist job using a distfile that lists all the libraries that are replicated. The rdist job checks for discrepancies, propagates any changes, and notifies the Netlib maintenance staff of any such changes.

\section{Email Netlib Server Installation}

The Netlib email server software is available from the Netlib repository itself. The software provides mechanisms for processing user email requests, which may involve keyword searching and file retrieval. The Netlib email server is installed at UT/ORNL and a number of other sites for the purpose of distributing numerical software. The software can be adapted easily, however, to distribute other types of software. For example, slightly modified versions are in use to distribute statistical software from statlibetemper.stat.cmu.edu and by the TeX User Group to distribute TeX-related software from tuglib@math.utah. edu. Appendix A lists Netlib sites and sites known to be running the Netlib email server.

\subsection{Acquiring and Installing the Netlib Software}

CAUTION: The most common problems with the email interface to Netlib are corrupted mail addresses, network errors, and so on. You should be reasonably expert with email before installing the Netlib email server.

To obtain the Netlib email server software, send the following message to netlibcornl.gov:

send netlib from misc

Netlib will return a shar file with instructions at the beginning as to how it should be unbundled. This guide assumes that the software is unbundled in a directory named /netlib/admin. After unbundling, carry out the following steps:

1. Edit the call to chdir in bin/reply.c to point to the place where the source code is stored on your system. /netlib is assumed for illustration here.

2. Edit/netlib/admin/LIBS and /netlib/master/index to reflect what you are distributing. Items strictly for local users go in LIBS.1cl and index.1cl; edit function groupid() in reply.c to define "local users". If everything is public, groupid() can simply return 0 . As it currently stands, "local" addresses are those whose machines are in /netlib/admin/groups/local; if any machine name in groups/enemies is found, processing is aborted.

Suppose you have a library eispack that you want to install. Make the directory /netlib/eispack; copy in files such as rg.f; create a file index there; add some lines to/netlib/admin/LIBS like

eispack $\Rightarrow$ eispack

eispac $\Rightarrow$ eispack 
(That second line is to allow for misspellings; use your imagination and watch the logfiles for common mistakes.) Be sure that the line

master $\Rightarrow$ master

is in LIBS, so that people can get the main index.

3. Edit the various disclaimers in /netlib/admin/mess. You may also wish to add disclaimer files in the source directories.

To activate mail processing:

* if you run 9th Edition UNIX, put

Pipe to /netlib/admin/bin/reply

in /usr/spool/mail/netlib and make that file owned by "netlibd", and similarly put

Pipe to /netlib/admin/bin/netlibd

in /usr/spool/mail/netlibd and make that file owned by yourself;

* if you run BSD UNIX, put

netlib: "|/netlib/admin/bin/reply"

netlibd: "//netlib/admin/bin/netlibd"

into/usr/lib/aliases and execute newaliases;

* else if your system has no equivalent mechanism, try the

daemon in /netlib/admin/bin/old-mail-sys.

The script admin/bin/netlibd contains (on line 3) cd /netlib, which you may need to change. Because Berkeley's alias facility provides no way to set the userid, you probably should put your name and address in the message so people know who is actually sending the mail.

To try the system out, echo send index | mail netlib and expect return mail in a couple minutes. A line should be added to /netlib/admin/log and admin/stderr should remain empty.

Once the basics are working, you can polish things a bit.

- Set up a nightly process to run /netlib/admin/bin/mkdirectory. Using the lo command (with source in bin/10.src) and adapting bin/mkfile you can create "dependencies" files in each Netlib directory to represent the relationship between source files there. Not only is this file itself useful to browsers, but by changing the lines in LIBS to the form

$$
\text { eispack } \Rightarrow-1 \theta i s p a c k
$$

you can teach Netlib to respond to a request for $\mathrm{rg}$ from eispack by sending not just $\mathrm{rg} . f$, but also balbak.f, hqr2.f, and so on. To reduce the size of the basic netlib distribution, the 10 source (all written by David Gay at Bell Labs) is now obtained by send lo from misc. 
- You should permanently save /netlib/admin/log so that bug fixes can be distributed, traffic measurements made, and annual summaries sent to code authors. The format of the log is: date time [address] bytes-sent library/item possibly followed by: $L=$ list,$F=$ find. The [address] is followed by "l" if the address was recognized as local. In contrast, the copy of incoming messages kept in /tmp/netreq is for debugging mail headers and monitoring illegal request syntax. Discard when convenient (perhaps by an $\mathrm{rm}$ in /etc/rc) or, if you prefer, comment out the line in reply.c:handle() that writes the file.

NOTE: There are a few things in reply.c, such as index/strchr and time.h, that aren't standard across different versions of UNIX. We have tried to deduce which system you run and generate appropriate code. Send reports of failures to Eric Grosse at ehgeresearch.att.com.

\section{NA-NET Database Setup and Maintenance}

\subsection{Acquiring the NA-NET Software}

The NA-NET software in use at UT/ORNL is not yet in general release. For more information about a possible future release date, send email to nanetena-net.ornl.gov.

\subsection{The NA-NET Program}

The NA-NET program handles the following:

- receipt of incoming mail for all NA-NET recipient addresses, including ordinary subscriber addresses (e.g., na.joe) and special functions (e.g., na.help, na.join, na.whois, etc.)

- receipt of bounced mail from attempts to send na-digests. These failed attempts are logged.

The NA-NET program is invoked by a modified version of sendmail whenever the modified sendmail receives a message addressed to: <something> Ona-net.ornl.gov. Other machines know to send mail addressed to na-net.ornl.gov to netlib2.cs.utk.edu because the latter machine is registered as the "mail exchanger" for na-net.ornl.gov with the Internet Domain Name System.

\subsection{NA-NET Files}

The NA-NET programs and files are in the directory/usr/local/na-net. The NANET main program is in /usr/local/na-net/na-net. The NA-NET program gets the location of all files from a config file that is passed to NA-NET on the UNIX command line when NA-NET is called by sendmail to deliver mail. The config file is in /usr/local/na-net/config. It contains the following: 


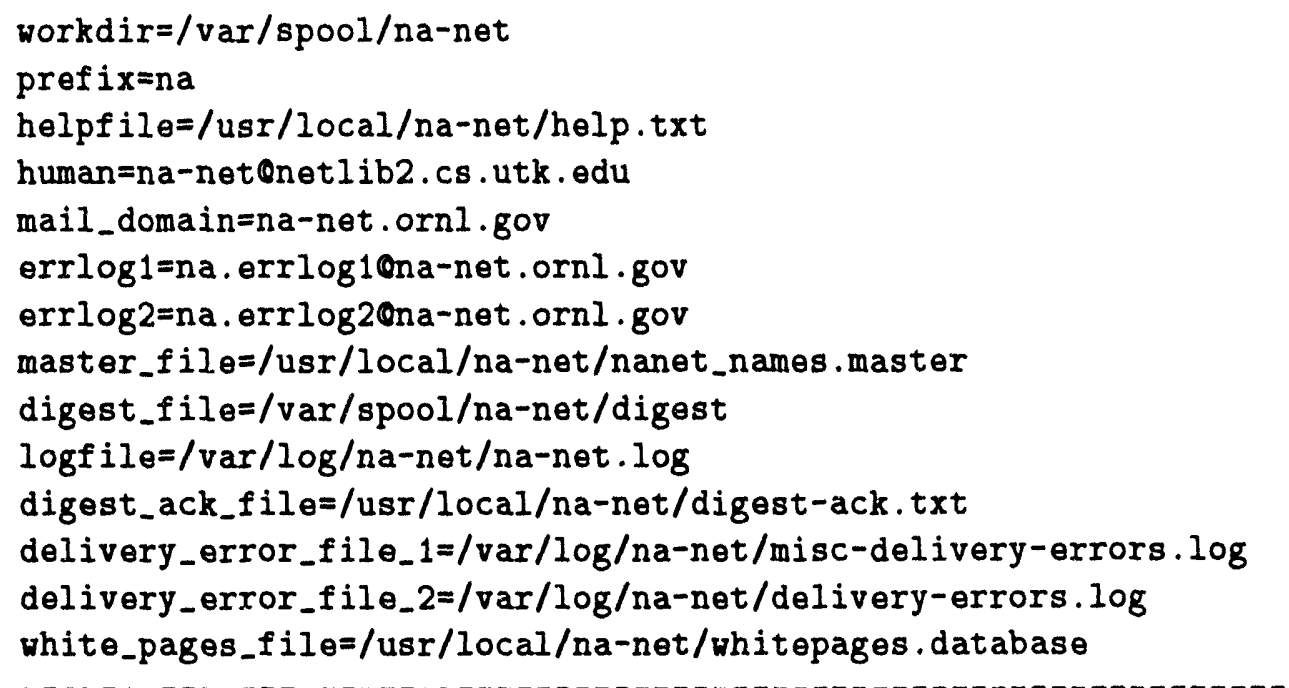

The purpose of these entries is as follows:

libdir - location of subsidiary programs to be called by NA-NET. (currently unused) workdir - a scratch directory for NA-NET to write temporary files, etc.

prefix - This is the prefix that NA-NET will accept at the beginning of addresses. NA-NET will remove it from the local part of a recipient address before processing.

The prefix is used to distinguish NA-NET addresses from ordinary local addresses on systems where the NA-NET system shares a mail domain with local users.

Normally the prefix doesn't change. However, you could run multiple NA-NET domains with different prefixes, simply by prefixes, simply by having sendmail call NA-NET with a different config-file for each prefix.

helpfile - location of the text file sent in response to a message addressed to help

human - Internet email address (not an NA-NET address) of a human being. This is used for mail to any of the following:

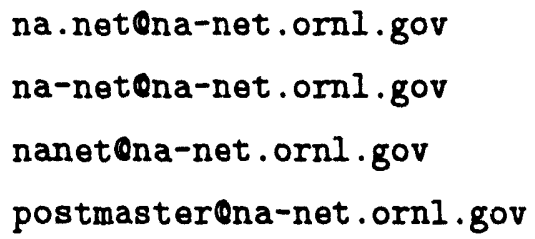

It is also used as a reply address on responses to help, join, change, remove, sendlist, whois, join-wp, change-wp, and remove-wp commands, and digest submissions. (But not as the reply address on digests sent to subscribers!) 
mail_domain -

Internet mail domain for NA-NET addresses. This is used in error messages and also so that NA-NET will recognize addresses such as user\%na-net.ornl.govena-net.ornl.gov which shouldn't occur, but do.

err $\log 1$ - This address is used for the return address on most responses to NA-NET commands. If a response bounces, the message will be sent back to this address. Normally errlog1 is set up to feed back into into errlog10na-net.ornl.gov, so that we can save the returned message. Unfortunately, if one of these response messages bounces, we can't do much about it; but the logs are sometimes useful to help answer questions or diagnose problems with someone's mail system. (See delivery_error_file_1, below).

err $\log 2$ - This should be an Internet email address. NA-NET will use this address as the envelope return-address on all outgoing na-digests. Normally this will point back to 〈prefix>.errlog20<mail_domain>, which allows NA-NET to process and $\log$ bounced mail messages. (Unlike messages sent in response to commands, we can use the bounced digests to let us know whose addresses are no longer valid.) (See delivery_error_file_2, below).

masterfile - location of the NA-NET subscriber database. See "File Formats" below.

digest file - This is where incoming digest articles are stored. Each article is separated from the others by a line of the form "From sender date". Two blank lines are added before the "From" line and after each message.

logfile - This is a log of messages sent to NA-NET. Each line is of the form:

date time sender recipient status-code status text...

digest_ack_file - This file contains a message that is sent to people who send mail to the na.digest.

delivery_errorfile_1 - This is where bounced messages in response to NA-NET responses get filed.

delivery_errorfile_2 - This is where bounced digests get filed.

whitepages file - location of the NA-NET Whitepages Database.

\subsection{File Formats}

1. na-digest subscriber database

The subscriber database is an ordinary text file consisting of lines of the form

lastname, firstname (na.key) email-address

These lines are sorted lexicographically by lastname then firstname. 
2. Whitepages Database

The format of the Whitepages Database is as follows:

1. Each record is separated from the next by a newline.

2. Each field of the record is separated from the next field by a CR (carriage return, control-M)

3. Any newlines within a field are represented as control-A.

It's difficult to read the file, but if the need arises it can be edited with a text editor.

The fields are, in order:

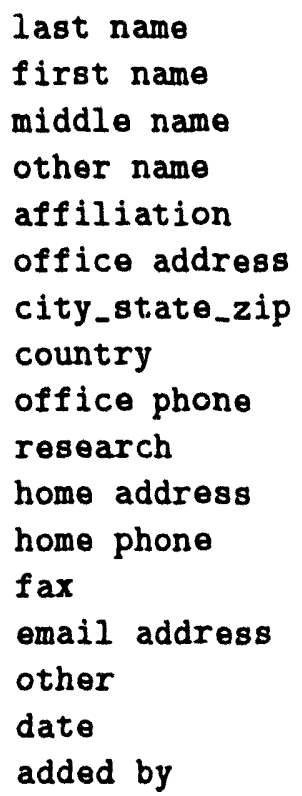

\subsection{NA-NET Source Files}

The source files are in /usr/local/src/na-net. Simply typing make should rebuild them.

Notes of any changes are in the file ChangeLog. The software is currently stable, but changes are made occasionally to fix (hopefully minor) bugs, to make the software more tolerant, easier to use, or easier to maintain.

\subsection{Database Changes, Backups, and Cron Jobs}

Database updates are currently performed by sequentially copying the nanet_names master or whitepages _database files to "new" files (those ending in .new). If the file copy happens successfully, the current database is linked to 
〈filename〉.01d and the new file is renamed to nanet names.master or whitepages database, respectively. The previous version of the file remains in 〈filename〉.old.

The new files are locked during updates so that two concurrent writes cannot happen, though others can read the database while it is being updated. The actual update - replacement of the old database file with the new - is atomic; queries can never see an inconsistent copy of the database file.

In order to prevent race conditions or locking the database for an excessive period of time, some operations may have to be backed out. For example, if someone tries to delete a subscriber record using the first and last names of the subscriber, and more than one subscriber has those names. In this case NA-NET will detect the condition, issue an appropriate message, and discard the new copy of the database (which may already have some records deleted) instead of replacing the old file. (Thus, the presence of a new file does not mean that file contains more current information.)

In addition to the normal system backups, and the .01d files, there is a cron job (/usr/local/na-net/rotate.sh) that gets run once per day that saves an extra copy of the NA-NET databases. Currently these are kept for five days.

A summary of NA-NET activity for a given day is run from cron at $11: 59 \mathrm{pm}$. The summary script is in (/usr/local/na-net/summary.sh)

There is currently no mechanism for rotating $\log$ files.

\subsection{Sending to Digest Subscribers}

Digest mailings are accomplished by sending a message to a special NA-NET address known to the digest moderator. Replies to digests are currently sent to the na.digest address.

\subsection{Surgery}

If it is necessary to change NA-NET databases "by hand", the NA-NET system should be suspended. In the directory /usr/local/na-net there is a script called na-net.sh; this does nothing but exit with a "temporary failure" status. If the normal NA-NET binary is moved aside and the na-net.sh file put in its place, sendmail will queue any messages for NA-NET and keep trying every half hour or so. After renaming the na-net.sh file, wait a few minutes so that any NA-NET processes can finish up before editing the database. After making whatever changes are necessary, don't forget to rename the real NA-NET program back to na-net.

\section{Installation and Customization of the Xnetlib Client}

\subsection{Acquiring the Xnetlib Software}

To acquire the Xnetlib client software from Netlib send email to netlibcorn1.gov with the line

send xnetlib.shar from xnetlib

in the message. Netlib will return the xnetlib.shar file by email. 
Xnetlib is available by anonymous FTP from netlib.ornl.gov in the xnetlib directory.

\subsection{System Requirements}

Xnetlib will build on nearly any UNIX system. It runs under the $X$ Window System, version 11 , from MIT. It requires release level 4 or greater and the Athena widget libraries as supplied by MIT.

Xnetlib is known to run on the following systems:

- Convex.

- DECStation running Ultrix 4.1, 4.2, and 4.2A, and DecWindows. (For Xnetlib to compile with the vendor-supplied $X$ Windows libraries, you must have the "Unsupported X11 Components" software subset loaded.) Xnetlib should also build and run with MIT's X11R4 or X11R5, but this configuration has not been tested.

- HP 9000 and MIT's X11R5. (Xnetlib may work with the vendor-supplied X Windows libraries, but this is not recommended.)

- IBM RS/6000 running AIX 3.1, 3.2, and MIT's X11R5. (Xnetlib may work with $\mathrm{X} 11 \mathrm{R} 4$, but $\mathrm{R} 5$ is preferred.)

- NeXT Dimension and Co-Exist X11R4.

- Sequent Symmetry and MIT's X11R4.

- SGI 4D/25 running IRIX 3.3.3 and X11R4.

- Stardent (Kubota) Titan and vendor-supplied X11R4.

- Sun 3 running SunOS 4.1 and X11R4.

- Sun 4 running SunOS 4.1 or later and X11R4 or X11R5.

\subsection{Building $X$ netlib}

Xnetlib makes use of the imake facility (via xmkmf) that comes with standard X Windows distributions. A generic Makefile is provided and can be used if imake is not present on your system.

After the executable is built, install it by copying the file xnetlib to an appropriate directory. There is no application defaults file to install.

With imake (xmkmf), type

$$
\text { xmkmf }
$$

make

Without imake (xmkmf), first edit Makefile.std to reflect your installation and then type

make -f Makefile.std 


\subsection{Customization of Xnetlib}

\subsubsection{Resources}

publicData - When a user runs Xnetlib, the index files for the libraries are downloaded from the server and cached in a special directory. For sites where many people use Xnetlib, it will save disk space if users share these index files. This can be accomplished by setting the xnlPath resource to some commonly writable directory and by setting the publicData resource to True. The effect of the publicData resource is to cause all index files to be saved world writable so they can be updated by anyone. Some sites set these defaults at compile time by setting the fallback resources for these variables. (Look for fallback_resources in main.c.)

indexlifetime - controls frequency of client to server communication. This resource sets the number of days an index file will be used before Xnetlib checks with the server to find out if the file is out of date. The default value is seven days.

hostfilo - sets the name of host file, which contains the lists of servers to contact. The default is \$HOME/.xnetlibHosts.

email - specifies the Internet email address of the user. Xnetlib will attempt to guess the email address but will very often be wrong. The email address is recorded in the server's $\log$ and is used to inform users of software bugs and updates.

print Command - sets the format string of the print command. It should contain a "\%s" which is replaced when the command is executed by the name of the temporary file used in printing the text. The default is "lpr \%s".

showthoInfo-determines whether instructions for adding your name to the NA-NET Whitepages Database are shown in Who mode. The default is True.

entrySep - sets the string to be displayed between entries in Who mode. The default is "._...".

xn1DownloadPath - sets the directory where files selected for downloading are to be placed. The default is \$HOME/xnlFiles.

depcheck - sets the default value for dependency checking in Download mode.

confirmQuit - If the confirmQuit resource is True, you will be asked for confirmation before quitting Xnetlib.

dontWarp - turns off automatic cursor positioning if True.

okColor, badColor, and cautioncolor - affect the status message window background. The defaults are green, red, and yellow.

dial, needle, ef, and gasGauge.background - affect features of the gas gauge in Download mode. The defaults are DarkViolet, yellow, red, and white. 


\section{Installing and Running Nlrexecd}

Nlrexecd is the service provider daemon for the Xnetlib services. The nlrexecd daemons running at U'T/ORNL currently provide access to the Netlib software and document repository, the NA-NET Whitepages Database, the Performance Database, and the Conferences Database. Nlrexocd is written to be a general service provider, and can be configured to offer an arbitrary set of services that understand the Xnetlib protocol. A new service can be added easily by providing the code for the function to be called when the service is invoked.

\subsection{Acquiring the Nlrexecd Software}

Nlrexecd is not yet in general release. Send email to xnetlibocs.utk.edu for more information.

\subsection{System Requirements}

Nlrexecd should build and run on any UNIX system supporting TCP/IP domain sockets. Nirexecd does not require any type of $\mathrm{X}$ Windows support.

\subsection{Building Nlrexecd}

There are two flavors of Nlrexecd. The large server distribution provides file transfer and keyword and database lookup and is the basis for the main Xnetlib server. The small server distribution provides only the file transfer service. Site-specific services can easily be added to either server.

Building the small server requires a file called nlrexecd_small.tar and otherwise requires a file called nlrexecd.tar.

Untar the file in a suitable area. Examine the Makefile and make any site-specific changes you may require. Type make to build nlrexecd.

Modules used by the small server are also used by the large server. Code that is specific to one or the other within these common modules is differentiated by the SMALL_SERVER preprocessor symbol.

\subsection{Services and Protocol}

All actions performed by nlrexecd are indicated by a unique service name. The service required is passed by name to nlrexecd after a TCP connection is established from the client. 


\begin{tabular}{|l|l|}
\hline & Xnetlib Protocol \\
\hline server (nlrexecd) & client (xnetlib or telnet) \\
\hline listens on TCP port 5555 & $\begin{array}{l}\text { opens TCP connection to server } \\
\text { sends newline terminated email address } \\
\text { sends newline terminated service name } \\
\text { sends newline terminated service specific data }\end{array}$ \\
$\begin{array}{l}\text { service specific internal call } \\
\text { is made passing client } \\
\text { data and socket descriptor }\end{array}$ & \\
\hline
\end{tabular}

The nlrexecd protocol describes only what occurs up until the service name and data are correctly specified, after that point the connection is "taken over" by that service.

Note that requiring the client to provide any service specific data is a violation of the spirit of separation between the service and the nlrexec layer. It was done entirely to simplify the job of the service module writer. There are several instances in which the service simply ignores this data. In any case, it may not be omitted by the client.

\subsubsection{Adding a Service to Nlrexecd}

Adding a new service to the server code involves writing the function to be called when your service is invoked and adding the service name in the main module. The following example shows how to add a service called "howdy".

To add the service name in the main module edit the file nlrexecd.c.

1. Declare your function (which should return a char*) where the other service functions are declared.

char *howdy();

2. Add the name of your service to the service 1 ist structure.

"howdy",

3. Add the name of your function to the service_call structure.

howdy,

Notice that the position of "howdy" in service.list should correspond to the position of howdy in service_call.

4. Create a new file called howdy.c. It should look something like this.

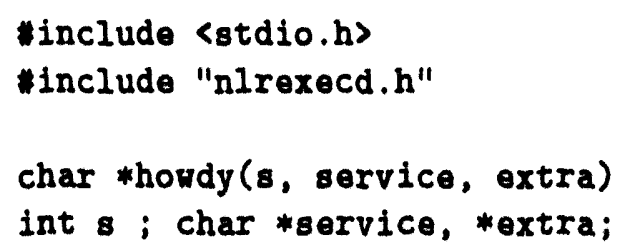




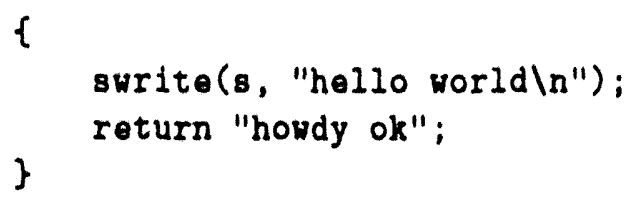

The string returned by a service function is written to the $\log$ file.

5. Add howdy . 0 to OBJS in the Makefile.

Now type make.

To test your new service start the server and use telnet to talk to it.

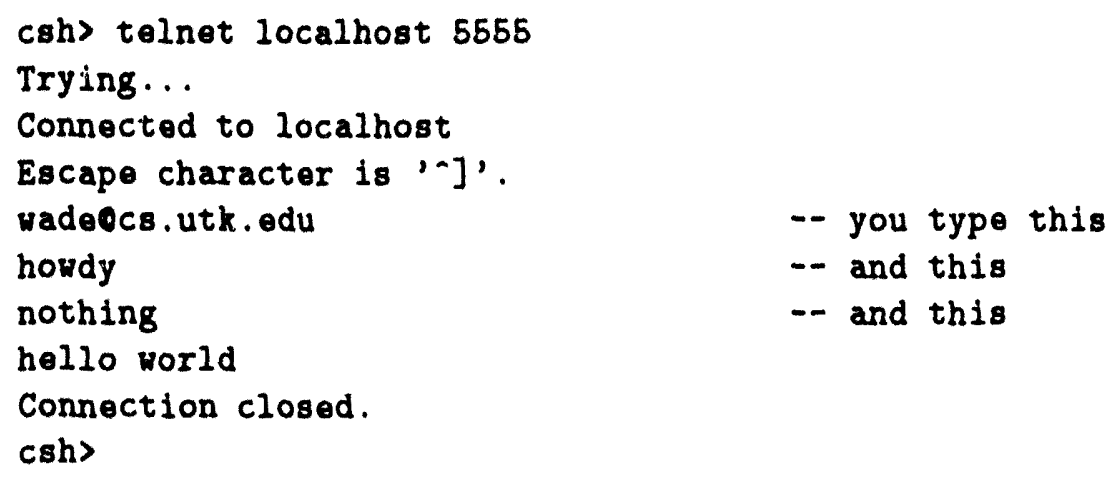

\subsubsection{Reserved Service Names}

To avoid service name conflicts it is intended that Xnetlib service name prefixes will be maintained in a central registry. Entities will be provided a service name prefix that they can use to manufacture unique service names. This would work in a manner similar to the Domain Name System except with the most general qualifier at the beginning of the name instead of the end.

\begin{tabular}{|c|c|}
\hline \multicolumn{2}{|c|}{ Reserved Service Names } \\
\hline $\begin{array}{l}\text { list-services } \\
\text { ? }\end{array}$ & required by all servers \\
\hline $\begin{array}{l}\text { file-tag } \\
\text { file-get } \\
\text { file-get-dep }\end{array}$ & used by all servers \\
\hline $\begin{array}{l}\text { who } \\
\text { keyword } \\
\text { keyword-or } \\
\text { keyword-and } \\
\text { keyword-lsi } \\
\text { keyword-literal } \\
\text { keyword-literal-case }\end{array}$ & used by large server \\
\hline $\begin{array}{l}\text { performance } \\
\text { dataserve } \\
\text { f2c }\end{array}$ & reserved prefixes \\
\hline
\end{tabular}


9.5. Command Line Options

\begin{tabular}{l|l|l} 
Option & Explanation & Default \\
\hline -port PORTNUMBER & $\begin{array}{l}\text { port at which nlrexecd will } \\
\text { listen for client requests }\end{array}$ & 5555 \\
\hline -dir DIRECTORY & $\begin{array}{l}\text { effective root directory } \\
\text { (argument to chroot) }\end{array}$ & "/notlib" \\
\hline -nochroot & Don't do chroot & \\
\hline -log PATHNAME & pathname for log file & "/usr/local/logs/nlr.10g"
\end{tabular}

\subsection{Keyword and Database Lookup Services}

\subsubsection{Keyword Lookup}

The keyword lookup services keyword, keyword-or, and keyword-and use an ndbm keyword database that is manufactured nightly from the netlib index files. This database is a full-text index and includes all words in the netlib index files except those that are excluded by a common word list filter.

The keyword-literal and keyword-literal-case searches are slower because they do string matching on the index file descriptions themselves.

The keyword searching mechanisms are expected to be changed to use WAIS indexing and searching in the near future.

\subsubsection{Latent Semantic Indexing}

Latent Semantic Indexing is a method for automatic indexing and retrieval that tries to take advantage of the semantic, or conceptual, content of documents. The particular LSI technique used in Xnetlib at UT/ORNL employs singular-value decomposition (SVD) to take a large matrix of term-document association data (in the case of Xnetlib, the documents are the netlib index files) and construct a "semantic" space wherein terms and documents that are closely associated are placed near one another. LSI tries to tackle the problems of synonymy (many ways to refer to the same object) and polysemy (more than one meaning for a term), so as to improve the recall and precision of retrieval. In fact, terms that do not actually appear in a document may still end up close to the document, if that is consistent with the major patterns of association in the data. Retrieval is carried out by using the terms in a query to identify a point in the semantic space and by returning documents in the neighborhood of this space. At UT/ORNL, the SVD is done periodically on a matrix constructed from the netlib index files to produce a semantic space for the netlib repository. The keyword-1si service invoked from nlrexecd carries out retrieval. For more information about LSI, see [3].

The particular LSI technique currently used in Xnetlib is patented and proprietary and can be used only with the written permission of Bell Communications Research.

\subsubsection{Whois Service}

The nlrexecd whois service does a lookup on the NA-NET Whitepages Database, augmented by the SIAM Membership List. Nlrexecd opens the file 
/netlib/whois/whitepages.database and does a linear search for matches to the user's query.

\subsubsection{Performance Database Service}

The Performance Database service uses the public-domain RDB relational DBMS developed by Walter Hobts of Rand Corporation. RDB tables are stored as regular UNIX ASCII files and thus can be manipulated by the normal UNIX utilities. The RDB tables for UT/ORNL Performance Database are stored in the performance library in the Netlib tree. The performance-query, performance-or-search, and performance-and-search services invoked from nlrexecd carry out searches on the RDB tables.

\subsubsection{Conference Database Service}

The Conference Database service currently uses the Postgres extended relational database system. Postgres is in the public domain and is available via anonymous FTP from the University of California at Berkeley. The function called by nlrexecd for this service is dataserve. dataserve takes a database-system-independent client request and translates it into the appropriate Postquel language queries which are then executed on the Postgres database.

The conference description files are stored in the confdb library in the netlib tree. The filenames for these descriptions are the Postgres Object IDs foi the corresponding entries in the Postgres database. Although the descriptions themselves are not stored in Postgres, a full-text index derived from these descriptions is stored in a Postgres relation. Postgres is used to handle searching by dates, keywords, and location. A geographical database stored in a Postgres relation handles geographical aliasing and hierarchical geographical relationships (e.g., retrieving entries for Belgium when asked for those in Europe).

\section{Anonymous FTP Server for Netlib}

An anonymous FTP server has been installed on netlib2.cs.utk.edu to provide anonymous FTP access to the Netlib directory tree. The Netlib anonymous FTP server is based on the Washington University ftp daemon, which in turn is based on the 4.3-Reno BSD ftp daemon. Minor modifications were necessary to get it to compile in a vanilla SunOS environment. The server supports several useful features including per-site, per-user, and per-directory access control, extensive logging, automatic display of "readme" messages when you cd to a particular directory, and automatic creation of compressed and/or tarred versions of files and directories. (Just ask for 〈filename $\rangle. Z$ instead of filename, or 〈directory>.tar. $Z$ instead of each file in the directory, and it gets packaged up on-the-fly.)

In "anonymous" mode, ftp logs into the "ftp" account and changes its root directory to that account's home directory, so that it becomes impossible to access files outside of that directory. On netlib2, the "ftp" account's home directory is /netlib.

Configuration files are as follows: 
/etc/ftpusers

This file contains a list of users (like "nobody") who are not permitted to $\log$ in via ftp.

/usr/local/etc/ftpaccess

This file specifies who can use the ftp server

(you can create classes based on where someone logs in

from and who they say they are), how many members of each

class can $\log$ in at once, which files get printed out

when you cd to a particular directory, a message to be

displayed at login time, whether a class of user can

request auto compression or auto-tar, what kinds of things

can be logged, who can "upload" files, and where warning

messages get mailed.

See "man ftpaccess" for more information.

/usr/local/logs/ftpd.log

This is where transactions get logged.

There are other config files, detailing other features of $\mathrm{ftp}$, but they are not used by the netlib implementation.

For more information, see the man pages for ftpaccess (5), ftpconversions(5), ftpcount (1), ftpd (8), ftphosts (5), ftpshut (8), ftpwho(1), and xferlog (5). (On netlib2, these man pages are installed in /usr/local/man; make sure your MANPATH environment variable contains/usr/local/man ahead of /usr/man).

Netlib's ftp server is installed in/usr/local/etc/ftpd. The Sun-supplied binary is in /usr/etc/in.ftpd; but the file/etc/inetd.conf has been changed to point to /usr/local/etc/ftpd.

A few other programs are also needed to make anonymous FTP work properly special (statically-linked) versions of Is, compress, and (GNU) tar. These are installed on netlib2 in /netlib/bin. On netlib2 there is also a dummy /netlib/etc/passwd file, which contains dummy entries for root and ftp - with fake passwords. These are so that the output of $1 \mathrm{~s}-1$ can use meaningful user names rather than uid numbers.

The sources are in /usr/local/src/wu-ftpd-2.1a. There are some documentation files there that detail how to configure it. For these instructions see /usr/local/src/wu-ftpd-2.1a/INSTALL and /usr/local/src/wu-ftpd-2.1a/NOTES. 


\section{Netlib Anonymous RCP Implementation}

\section{1. "anon" Account}

An "anon" account exists on the machines netlib1.epm.ornl.gov and netlib2.cs.utk.edu for the purpose of allowing anonymous RCP access. Normal logins to this account are disabled by giving it a passwd field of "*". In addition, the shell for this account is a special shell named "anon-shell".

\subsection{Invocation of Anonymous RCP}

The remote client's rcp command invokes the rcp command on the netlib server machines via the remote shell service (rshd). rshd on the Netlib machines has been specially modified to accept the following syntax in .rhosts files:

- If the remote-user field is "*", and the remote-machine field is filled in, any remote user at that machine can execute commands.

- If the remote-machine field is "*", and the remote-user field is filled in, any user by that name on any machine can execute commands.

- If both fields are "*", anyone can execute commands for this particular user.

The .rhosts file for user anon on the netlib machines currently consists of the following line:

The rshd program defines the environment variables REMOTE_HOST and REMOTE_USER for use by programs that it runs.

\section{3. anon-shell}

"anon-shell" is a very primitive command parser. Basically, it understands c-shell style quoting and globbing (wildcard expansion). When given a command, it splits it up into arguments, expands wildcards on each argument, and then attempts to execute that command. It has a built-in table of commands that it will attempt to run. It will refuse to run any commands that are not in its hard-coded table.

"anon-shell" also logs every command executed, along with the remote user and host, via the syslog facility. Currently it uses LOG_DAEMON and LOG_INFO. netlib2's syslog currently stores such entries in /usr/adm/anon-rcp-log.

"anon-shell" currently has two commands: rcp and ls. rcp is used by the client rcp program to retrieve remote files. Is can be used to browse directories.

IMPORTANT NOTE: "anon-shell" runs set-uid to root and passes root privilege to any commands that it runs. Thus, it is dangerous to add new commands without going over them carefully. 


\subsection{Modified rcp and is commands}

The versions of rcp and ls installed on netlib1.epm.ornl.gov and netlib2.cs.utk. edu have been modified as follows:

- Both rcp and is immediately do the following:

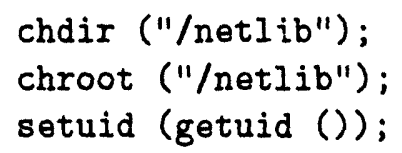

thus limiting their view of the world to everything under /netlib, and turning off any special privileges.

- The modified rcp can deal with not having an /etc/services or /etc/passwd file.

- The modified rcp has all calls to mkdir( $)$ and open $(\ldots, 0$ CREAT,$\ldots$ \#ifdefed out and replaced with code that prints "Permission denied". In general, the file receiving code is disabled, but it will talk protocol with the client rcp and return error messages. The file sending code works normally.

- rcp and ls are statically-linked binaries, since they have no access to system shared libraries.

\subsection{Locations of files}

The source to the modified rsh program is in the directory /usr/local/src/rsh.

The specially modified rshd program is installed in /usr/etc/in.rshd. (the original one is in /usr/etc/in.rshd.ORIG)

The sources to the other commands are in /usr/local/src/anon-rcp and its subdirectories. The subdirectories $1 \mathrm{~s}$, shell, and $\mathrm{rcp}$ contain the sources to anon-1s, anon-shell, and anon-rcp. These are installed in anon, which is currently /usr/local/homes/anon.

Logs of transactions are currently kept in /var/adm/anon-rcp-log. 


\section{A. Netlib Sites}

$\begin{array}{ll}\text { A.1. Sites Mirroring the Netlib Repository } \\ \text { New Jersey } & \text { netliberesearch.att.com } \\ \text { Tennessee } & \text { netlibeornl.gov } \\ \text { Norway } & \text { netlibenac.no } \\ \text { England } & \text { netlib@ukc.ac.uk } \\ \text { Germany } & \text { elibesc.zib-berlin.de } \\ \text { Taiwan } & \text { netlibenchc.edu.tw } \\ \text { Australia } & \text { netlibedraci.cs.uow.edu.au }\end{array}$

\section{A.2. Some Sites Using the Netlib Email Server to Distribute Other Types of Software}

The software that runs the Netlib email server is available from the Netlib repository. It can be retrieved by sending the message send netlib from misc to netlib.ornl.gov. A number of groups have acquired the email distribution software, and a few of these are listed below.

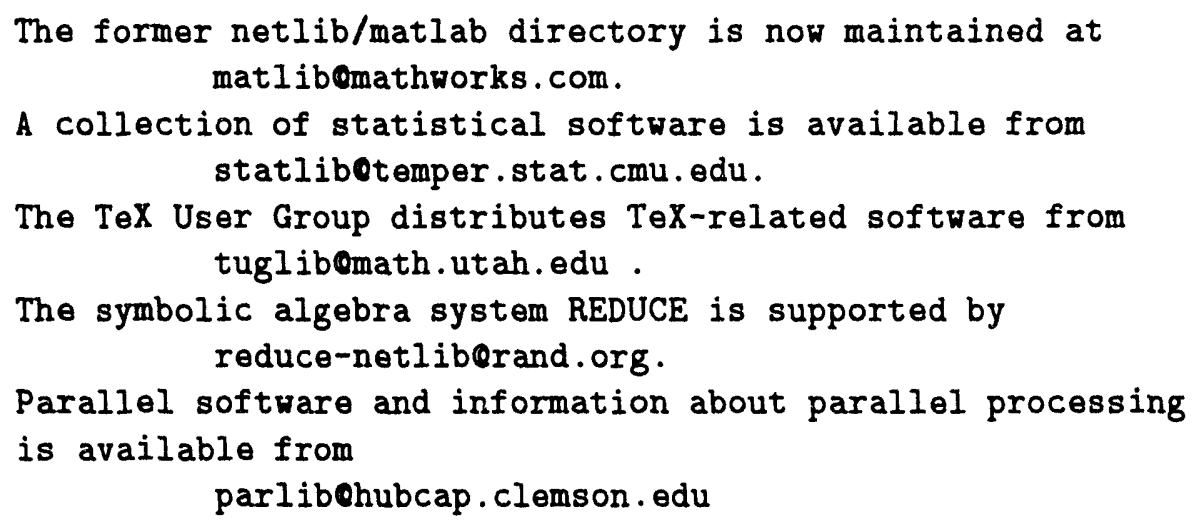

\section{References}

[1] Michael W. Berry, Jack J. Dongarra, and Brian H. Larose. PDS: A performance database server. Scientific Computing, 1994. (to appear).

[2] Ronald F. Boisvert, S. E. Howe, and D. K. Kahaner. The Guide to Available Mathematical Software problem classification system. Comm. Stat. - Simul. Comp., 20(4):811-842, 1991.

[3] S. Deerwester, S. Dumais, G. Furnas, T. Landauer, and R. Harshamn. Indexing by latent semantic analysis. Journal of the American Society for Information Science, 41(6):391-407, September 1990.

[4] Jack Dongarra, Tom Rowan, and Reed Wade. Software distribution using XNETLIB. Technical Report CS-93-191, University of Tennessee-Knoxville, June 1993. 
[5] Jack J. Dongarra and Eric Grosse. Distribution of mathematical software via electronic mail. Communications of the $A C M, 30(5): 403-407$, May 1987.

[6] George E. Forsythe, Michael A. Malcolm, and Cleve B. Moler. Computer Methods for Mathematical Computations. Prentice Hall, Inc., 1977.

[7] Eric Grosse. Repository mirroring. (submitted for publication), 1994.

[8] Adrian Nye and Tim O'Reilly. X Window System User's Guide. O'Reilly and Associates, Inc., 1993. 


\section{Index}

anonymous FTP, 18

acquiring Xnetlib by, 11

server, 36

anonymous RCP, 18

implementation, 38

anonymous RSH, 18

benchmarks, 3

Conferences Database, 4, 15 server, 36

contacts, 5

dependency checking, 13

Xnetlib, 31

downloading, 13

xnlDownloadPath, 31

index file

format, 20

index files, 20

Xnetlib, 15 indexLifetime, 15, 31

shared, 31

Latent Semantic Indexing, 35

LSI, 35

mirroring, 20

NA-NET, 4

email interface, 6

file formats, 27

files, 25

joining, 7

name, 6

News Digest, 7

software, 25

source code files, 28

Whitepages, 15, 35

Whitepages email interface, 8

netlib

email request syntax, 5

Netlib repository, 1, 3, 20

nlrexecd, 32

adding a service to, 33

Performance Database, 3,15

server, 36

Postgres, 4,36

RDB, 36

replication, 20, 22

searching, 15

netlibget, 17

SIAM membership list, 4,15

support, 5

TCP/IP, 10

user interface

anonymous, 18

command-line, 17

email, 5

$\mathrm{X}$ Window, 10

Whitepages, 15

$\mathrm{X}$ Window System, 10

Xdefaults, 16

Xnetlib, 10

$\mathrm{X}$ resources, 31

acquiring, 11, 29

installation, 12, 30

man page, 12

operation, 13

protocol, 32

reference card, 12

server, 32

services, 32

system requirements, 11,30 


\section{INTERNAL DISTRIBUTION}

1. B.R. Appleton

2. T.S. Darland

3. E.F. D'Azevedo

4. J.M. Donato

5-9. J.J. Dongarra

10. G.A. Geist

11. S.L. Lee

12. N. Nachtigal

13. E.G. $\mathrm{Ng}$

14. C.E. Oliver

15. P.M. Papadopoulos

16-20. S.A. Raby

21-25. T.H. Rowan
26-30. R.F. Sincovec

31-35. R.C. Ward

36. D.W. Walker

37. P.H. Worley

38. Central Research Library

39. ORNL Patent Office

40. K-25 Applied Technology Library

41. Y-12 Technical Library

42. Laboratory Records Department - RC

43-44. Laboratory Records Department

\section{EXTERNAL DISTRIBUTION}

45. Cleve Ashcraft, Boeing Computer Services, P.O. Box 24346, M/S 7L-21, Seattle, WA $98124-0346$

46. Donald M. Austin, 6196 EECS Bldg., University of Minnesota, 200 Union St., S.E., Minneapolis, MN 55455

47. Robert G. Babb, Oregon Graduate Institute, CSE Department, 19600 N.W. von Neumann Drive, Beaverton, OR 97006-1999

48. Lawrence J. Baker, Exxon Production Research Company, P.O. Box 2189, Houston, TX 77252-2189

49. Jesse L. Barlow, Department of Computer Science, Pennsylvania State University, University Park, PA 16802

50. Edward H. Barsis, Computer Science and Mathematics, P.O. Box 5800, Sandia National Laboratories, Albuquerque, NM 87185

51. Chris Bischof, Mathematics and Computer Science Division, Argonne National Laboratory, 9700 South Cass Avenue, Argonne, IL 60439

52. Ake Bjorck, Department of Mathematics, Linkoping University, S-581 83 Linkoping, Sweden

53. Jean R. S. Blair, Department of Computer Science, Ayres Hall, University of Tennessee, Knoxville, TN 37996-1301

54. Roger W. Brockett, Pierce Hall, 29 Oxford Street, Harvard University, Cambridge, MA 02138

55. James C. Browne, Department of Computer Science, University of Texas, Austin, TX 78712

56-60. Shirley V. Browne, Department of Computer Science, Ayres Hall, University of Tennessee, Knoxville, TN 37996-1301 
61. Bill L. Buzbee, Scientific Computing Division, National (enter for Almospherie Research, P.O. Box 3000, Boulder, CO 80307

62. Donald A. Calahan, Department of Electrical and Computer Engineering, University of Michigan, Ann Arbor, MI 48109

63. Ian Cavers, Department of Computer Science, University of British Columbia, Vancouver, British Columbia V6'I IW5, Canada

64. Tony Chan, Department of Mathematics, University of California, Los Angeles, 405 Hilgard Avenue, Los Angeles, CA 90024

65. Jagdish Chandra, Army Research Office, P.O. Box 12211, Research Triangle Park, NC 27709

66. Eleanor Chu, Department of Mathematics and Statistics, University of Ciuelph, Guelph, Ontario, Canada NIG 2WI

67. Melvyn Ciment, National Science Foundation, 1800 G Street N.W., Washington, DC 20550

68. Tom Coleman, Department of Computer Science, Cornell University, Ithaca, NY 14853

69. Paul Concus, Mathematics and Computing, Lawrence Berkeley Laboratory, Berkeley, CA 94720

70. Andy Conn, IBM T. J. Watson Research Center, P.O. Box 218, Yorktown Heights, NY 10598

71. John M. Conroy, Supercomputer Research Center, 17100 Science Drive, Bowie, MI) $20715-4300$

72. Jane K. Cullum, IBM T. J. Watson Research Center, P.O. Box 218, Yorktown Heights, NY 10598

73. George Cybenko, Center for Supercomputing Research and Development, University of Illinois, $104 \mathrm{~S}$. Wright Street, Urbana, IL 61801-2932

74. George J. Davis, Department of Mathematics, Georgia State University, Atlanta, GA 30303

75. Tim A. Davis, Computer and Information Sciences Department, 301 CSE, University of Florida, Gainesville, FL 32611-2024

76. Donald J. Dudziak, Department of Nuclear Engineering, 110B Burlington Engineering Labs, North Carolina State University, Raleigh, NC' 27695-7909

77. Lain Duff, Atlas Centre, Rutherford Appleton Laboratory, Didcot, Oxon OX11 OQX, England

78. Patricia Eberlein, Department of Computer Science, SUNY at Buffalo, Buffalo, NY 14260

79. Stanley Eisenstat, Department of Computer Science, Yale University, P.O. Box 2158 Yale Station, New Haven, CT 06520

80. Lars Elden, Department of Mathematics, Linkoping University, 58183 Linkoping, Sweden

81. Howard C. Elman, Computer Science Department, University of Maryland, College Park, MD 20742 
82. Albert M. Erisman, Boeing Computer Services, Engineering Technology Applications, ETA Division, P.O. Box 24346, MS-7L-20 Seatle, WA 98124-0346

83. Geoffrey C. Fox, Northeast Parallel Architectures Center, 111 College Place, Syracuse University, Syracuse, NY 13244-4100

84. Paul O. Frederickson, NASA Ames Research Center, RIACS, MIS T045-1, Moffett Field, CA 94035

85. Fred N. Fritsch, L-316, Computing and Mathematics Research Division, Lawrence Livermore National Laboratory, P.O. Box 808, Livermore, CA 94550

86. Robert E. Funderlic, Department of Computer Science, North Carolina State University, Raleigh, NC 27650

87. K. Gallivan, Computer Science Department, University of Illinois, Urbana, IL 61801

88. Dennis B. Gannon, Computer Science Department, Indiana University, Bloomington, IN 47405

89. Feng Gao, Department of Computer Science, University of British Columbia, Vancouver, British Columbia V6T IW5, Canada

90. David M. Gay, Bell Laboratories, 600 Mountain Avenue, Murray Hill, NJ 07974

91. C. William Gear, NEC Research Institute, 4 Independence Way, Princeton, NJ 08540

92. W. Morven Gentleman, Division of Electrical Engineering, National Research Council, Building M-50, Room 344, Montreal Road, Ottawa, Ontario, Canada K1A 0R8

93. J. Alan George, Vice President, Academic and Provost, Needles Hall, University of Waterloo, Waterloo, Ontario, Canada N2L 3G1

94. John R. Gilbert, Xerox Palo Alto Research Center, 3333 Coyote Hill Road, Palo Alto CA 94304

95. Gene H. Golub, Department of Computer Science, Stanford University, Stanford, CA 94305

96. Joseph F. Grcar, Division 8245 , Sandia National Laboratories, Livermore, CA $94551-0969$

97-101. Stan C. Green, Depa tment of Computer Science, Ayres Hall, University of Tennessee, Knoxville, TN 37996-1301

102. John Gustafson, Ames Laboratory, lowa State University, Ames, IA 50011

103. Per Christian Hansen, $\mathrm{UCI}^{*} \mathrm{C}$ Lyngby, Building 305 , Technical University of Denmark, DK-2800 Lyngby, Denmark

104. Richard Hanson, IMSL Inc., 2500 Park West Tower One, 2500 City West Blvd., Houston, TX 77042-3020

105. Michael T. Heath, National Center for Supercomputing Applications, 4157 Beckman Institute, University of Illinois, 405 North Mathews Avenue, Urbana, IL $61801-2300$

106. Don E. Heller, Physics and Computer Science Department, Shell Development Co., P.O. Box 481, Houston, TX 77001 
107. Nicholas J. Higham, Department of Mathematics, University of Manchester, Girt Manchester, M13 9PL. England

108. Charles J. Holland, Air Force Office of Scientific Research, Building 410, Bolling Air Force Base, Washington, DC 20332

109. Robert E. Huddleston, Computation Department, Lawrence Livermore National Laboratory, P.O. Box 808, Livermore, CA 94550

110. Ilse Ipsen, Department of Computer Science, Yale University, P.O. Box 2158 Yale Station, New Haven, CT 06520

111. Barry Joe, Department of Computer Science, University of Alberta, Edmonton, Alberta T6G 2H1, Canada

112. Lennart Johnsson, Thinking Machines Inc., 245 First Street, Cambridge, MA $02142-1214$

113. Harry Jordan, Department of Electrical and Computer Engineering, University of Colorado, Boulder, CO 80309

114. Bo Kagstrom, Institute of Information Processing, University of Umea, 5-901 87 Umea, Sweden

115. Malvyn H. Kalos, Cornell Theory Center, Engineering and Theory Center Bldg., Cornell University, Ithaca, NY 14853-3901

116. Hans Kaper, Mathematics and Computer Science Division, Argonne National Laboratory, 9700 South Cass A venue, Bldg. 221, Argonne, IL 60439

117. Linda Kaufman, Bell Laboratories, 600 Mountain Avenue, Murray Hill, NJ 07974

118. Robert J. Kee, Division 8245, Sandia National Laboratories, Livermore, CA 94551. 0969

119. Kenneth Kennedy, Department of Computer Science, Rice University, P.O. Box 1892, Houston, TX 77001

120. Thomas Kitchens, Department of Energy, Scientific Computing Staff, Office of Energy Research, ER-7, Office G-236 Germantown, Washington, DC 20585

121. Richard Lau, Office of Naval Research, Code 111MA, 800 Quincy Street, Boston Tower 1, Arlington, VA 22217-5000

122. Alan J. Laub, Department of Electrical and Computer Engineering, University of California, Santa Barbara, CA 93106

123. Robert L. Launer, Army Research Office, P.O. Box 12211, Research Triangle Park, NC 27709

124. Charles Lawson, MS 301-490, Jet Propulsion Laboratory, 4800 Oak Grove Drive, Pasadena, CA 91109

125. Peter D. Lax, Courant Institute of Mathematical Sciences, New York University, 251 Mercer Street, New York, NY 10012

126. James E. Leiss, Rt. 2, Box 142C, Broadway, VA 22815

127. John G. Lewis, Boeing Computer Services, P.O. Box 24346, M/S 7L-21, Seattle, WA $98124-0346$ 
128. Jing Li, IMSL Inc., 2500 Park West Tower One, 2500 City West Blvd., Houston, $\mathrm{TX} 7 \mathrm{i} 042-3020$

129. Heather M. Liddell, Center for Parallel Computing, Department of Computer Science and Statistics, Queen Mary College, University of London, Mile End Road, London El 4NS, England

130. Arno Liegmann, c/o ETH Rechenzentrum, Clausiusstr. 55, CH-8092 Zurich, Switzerland

131. Joseph Liu, Department of Computer Science, York University, 4700 Keele Street, North York, Ontario, Canada M3J IP3

132. Robert F. Lucas, Supercomputer Research Center, 17100 Science Drive, Bowie, MD 20715-4300

133. Franklin Luk, Department of Computer Science, Amos Eaton Building - \#131, Rensselaer Polytechnic Institute, Troy, NY 12180-3590

134. Thomas A. Manteuffel, Department of Mathematics, University of Colorado Denver, Campus Box 170, P.O. Box 173364, Denver, CO 80217-3364

135. Consuelo Maulino, Universidad Central de Venezuela, Escuela de Computacion, Facultad de Ciencias, Apartado 47002, Caracas 1041-A, Venezuela

136. James McGraw, Lawrence Livermore National Laboratory, L-306, P.O. Box 808 , Livermore, CA 94550

137. Paul C. Messina, Mail Code 158-79, California Institute of Technology, 1201 E. California Blvd., Pasadena, CA 91125

138. Cleve Moler, The Mathworks, 325 Linfield Place, Menlo Park, CA 94025

139-143. Keith Moore, Department of Computer Science, Ayres Hall, University of Tennessee, Knoxville, TN 37996-1301

144. Neville Moray, Department of Mechanical and Industrial Engineering, University of Illinois, 1206 West Green Street, Urbana, IL 61801

145. Dr. David Nelson, Director of Scientific Computing, ER-7, Applied Mathematical Sciences, Office of Energy Research, U.S. Department of Energy, Washington, DC 20585

146. Dianne P. O'Leary, Computer Science Department, University of Maryland, College Park, MD 20742

147. James M. Ortega, Department of Applied Mathematics, Thornton Hall, University of Virginia, Charlottesville, VA 22901

148. Charles F. Osgood, National Security Agency, Ft. George G. Meade, MD 20755

149. Chris Paige, McGill University, School of Computer Science, McConnell Engineering Building, 3480 University Street, Montreal, Quebec, Canada H3A 2A7

150. Roy P. Pargas, Department of Computer Science, Clemson University, Clemson, SC 29634-1906

151. Beresford N. Parlett, Department of Mathematics, University of California, Berkeley, CA 94720

152. Merrell Patrick, Department of Computer Science, Duke University, Durham, NC 27706 
153. Robert J. Plemmons, Departments of Mathematics and Computer Science, Box 7311, Wake Forest University, Winston-Salem, NC 27109

154. James C. T. Pool, Deputy Director, Caltech Concurrent Supercomputing Facility, California Institute of Technology, MS 158-79, Pasadena, CA 91125

155. Jesse Poore, Department of Computer Science, Ayres Hall, University of Tennessee, Knoxville, TN 37996-1301

156. Alex Pothen, Department of Computer Science, Pennsylvania State University, University Park, PA 16802

157. Yuanchang Qi, IBM European Petroleum Application Center, P.O. Box 585, N4040 Hafrsfjord, Norway

158. Giuseppe Radicati, IBM European Center for Scientific and Engineering Computing, via del Giorgione 159, 1-00147 Roma, Italy

159. John K. Reid, Numerical Analysis Group, Central Computing Department, Atlas Centre, Rutherford Appleton Laboratory, Didcot, Oxon OX11 0QX, England

160. Werner C. Rheinboldt, Department of Mathematics and Statistics, University of Pittsburgh, Pittsburgh, PA 15260

161. John R. Rice, Computer Science Department, Purdue University, West Lafayette, IN 47907

162. Garry Rodrigue, Numericai Mathematics Group, Lawrence Livermore Laboratory, Livermore, CA 94550

163. Donald J. Rose, Department of Computer Science, Duke University, Durham, NC 27706

164. Edward Rothberg, Department of Computer Science, Stanford University, Stanford, CA 94305

165. Axel Ruhe, Dept. of Computer Science, Chalmers University of Technology, S41296 Goteborg, Sweden

166. Joel Saltz, ICASE, MS 132C, NASA Langley Research Center, Hampton, VA 23665

167. Ahmed H. Sameh, Center for Supercomputing R\&D, 1384 W. Springfield Avenue, University of Illinois, Urbana, IL 61801

168. Michael Saunders, Systems Optimization Laboratory, Operations Research Department, Stanford University, Stanford, CA 94305

169. Robert Schreiber, RIACS, Mail Stop 230-5, NASA Ames Research Center, Moffet Field, CA 94035

170. Martin H. Schultz, Department of Computer Science, Yale University, P.O. Box 2158 Yale Station, New Haven, CT 06520

171. David S. Scott, Intel Scientific Computers, 15201 N.W. Greenbrier Parkway, Beaverton, OR 97006

172. Lawrence F. Shampine, Mathematics Department, Southern Methodist University, Dallas, TX 75275

173. Andy Sherman, Department of Computer Science, Yale University, P.O. Box 2158 Yale Station, New Haven, CT 06520 
174. Kermit Sigmon, Department of Mathematics, University of Florida, Gainesville, FL 32611

175. Horst Simon, Mail Stop T045-1, NASA Ames Research Center, Moffett Field, CA 94035

176. Anthony Skjellum, Lawrence Livermore National Laboratory, 7000 East Ave., L316, P.O. Box 808 Livermore, CA 94551

177. Danny C. Sorensen, Department of Mathematical Sciences, Rice University, P.O. Box 1892, Houston, TX 77251

178. G. W. Stewart, Computer Science Department, University of Maryland, College Park, MD 20742

179. Paul N. Swartztrauber, National Center for Atmospheric Research, P.O. Box 3000, Boulder, CO 80307

180. Philippe Toint, Dept. of Mathematics, University of Namni. FUNOP, 61 rue de Bruxelles, B-Namur, Belgium

181. Bernard Tourancheau, LIP, ENS-Lyon, 69364 Lyon cedex 07, France

182. Hank Van der Vorst, Dept. of Techn. Mathematics and Computer Science, Delft University of Technology, P.O. Box 356, NL-2600 AJ Delft, The Netherlands

183. Charles Van Loan, Department of Computer Science, Cornell University, Ithaca, NY 14853

184. Jim M. Varah, Centre for Integrated Computer Systems Research, University of British Columbia, Office 2053-2324 Main Mall, Vancouver, British Columbia V6T 1W5, Canada

185. Udaya B. Vemulapati, Dept. of Computer Science, University of Central Florida, Orlando, FL 32816-0362

186. Robert G. Voigt, ICASE, MS 132-C, NASA Langley Research Center, Hampton, VA 23665

187. Phuong Vu, Cray Research, Inc., 19607 Franz Rd., Houston, 'IX 77084

188-192. Reed C. Wade, Department of Computer Science, Ayres Hall, University of Tennessee, Knoxville, TN 37996-1301

193. Daniel D. Warner, Department of Mathematical Sciences, O-104 Martin Hall, Clemson University, Clemson, SC 29631

194. Robert P. Weaver 1555 Rockmont Circle Boulder, CO 80303

195. Mary F. Wheeler, Rice University, Department of Mathematical Sciences, P.O. Box 1892, Houston, TX 77251

196. Andrew B. White, Computing Division, Los Alamos National Laboratory, P.O. Box 1663, MS-265, Los Alamos, NM 87545

197. Margaret Wright, Bell Laboratories, 600 Mountain Avenue, Murray Hill, NJ 07974

198. David Young, University of Texas, Center for Numerical Analysis, RLM 13.150, Austin, TX 78731 
199. Earl Zmijewski, Department of Computer Science, University of California, Santa Barbara, CA 93106

200. Office of Assistant Manager for Energy Research and Development, U.S. Department of Energy, Oak Ridge Operations Office, P.O. Box 2001 Oak Ridge, TN $37831-8600$

201-202. Office of Scientific \& Technical Information, P.O. Box 62, Oak Ridge, TN 37831 

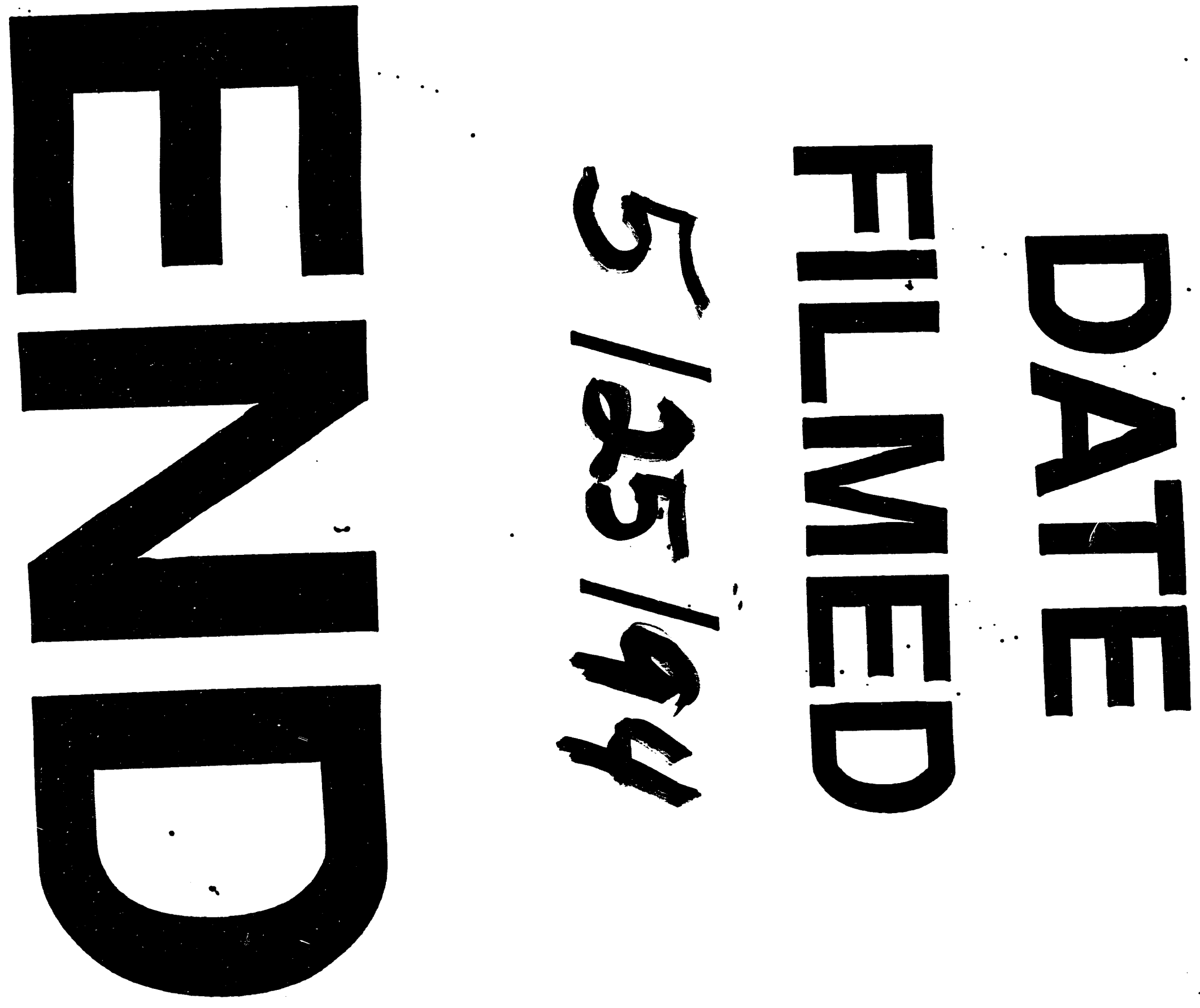
